\title{
Thermochemical convection in Earth's inner core
}

\author{
Renaud Deguen ${ }^{1,2}$ and Philippe Cardin ${ }^{2}$ \\ ${ }^{1}$ Department of Earth and Planetary Sciences, Johns Hopkins University, Baltimore, MD 21218, USA. E-mail: rdeguen@gmail.com \\ ${ }^{2}$ ISTerre, Université de Grenoble 1, CNRS, Grenoble, France
}

Accepted 2011 September 6. Received 2011 August 18; in original form 2011 May 16

\begin{abstract}
SUMMAR Y
The dynamics of Earth's inner core depends critically on whether it is stably stratified or unstably stratified. We propose here a general analysis of the thermal evolution of the inner core. Whether the geotherm in the inner core is superadiabatic or not depends on the inner core solidification rate, on the thermal diffusivity of iron at inner core conditions, and on the ratio of the Clapeyron slope to the adiabatic gradient in the inner core. The temperature field within the inner core can be destabilizing - and could drive convection - if the growth of the inner core is fast enough. The effect of radiogenic heating is probably small, and, perhaps surprisingly, can even stabilize the inner core against convection. The uncertainties are such that it is not possible at present to conclude about the likelihood of thermal convection in the inner core, but recent estimates of the Core-Mantle Boundary (CMB) heat flux and inner core conductivity favour convection. Thermal convection is more likely early in the inner core history, a consequence of the secular decrease in cooling rate of the core. In addition, solidification-induced partitioning of the light elements may induce a stable density stratification within the inner core.

We develop a numerical model of thermochemical convection in a growing inner core, which couples the evolution and dynamics of the inner core with the thermal and compositional evolution of the outer core. Melting and crystallization associated with deformation of the Inner Core Boundary (ICB) would be of importance for the style of convection if the viscosity is large, but we focus here on the case of low viscosity for which phase change associated with dynamic topography at the ICB is expected to play a secondary role. In this regime, convection is typical of high Rayleigh number internally heated convection, with cold plumes falling from the ICB.

Several possible scenarios can lead to a layered inner core, either because of cessation of thermal convection due to the decrease in cooling rate of the core, or because of a compositional stratification which can confine convection in the deep inner core, or stabilize the whole inner core. For each of these scenarios, it is possible to find plausible sets of parameters (inner core age, viscosity, magnitude of the compositional stratification) for which the radius at which convection stops corresponds to the radius of the seismically inferred innermost inner core.
\end{abstract}

Key words: Numerical solutions; Seismic anisotropy; Heat generation and transport.

\section{INTRODUCTION}

Over the years, the seismological picture of the inner core has become increasingly complex (e.g. Tkalčic 2010), with observed scales of heterogeneities and texture variations ranging from the inner core size to less than $1 \mathrm{~km}$ [see Souriau (2007) and Tkalcic $\&$ Kennett (2008) for recent reviews]. Some of these complexities may be artefacts due to pollution of the inner core signal by heterogeneities in D", but a coherent picture seems nevertheless to emerge. The presence of a cylindrical $\sim 3$ per cent anisotropy in $P$ wave velocity (Poupinet et al. 1983; Morelli et al. 1986; Woodhouse et al. 1986; Creager 1992; Tromp 1993) and attenuation (Souriau \& Romanowicz 1996, 1997) is well established, as is the East-West asymmetry in $P$-wave velocity, anisotropy and attenuation (Tanaka \& Hamaguchi 1997; Creager 1999; Garcia \& Souriau 2000; Niu \& Wen 2001; Garcia 2002; Cormier 2007). The degree of anisotropy increases with depth, the anisotropy being weak or non-existent in the upper 100-200 km of the inner core (Song \& Helmberger 1995; Garcia \& Souriau 2000). The presence of an innermost inner core of radius $300-600 \mathrm{~km}$, with a distinct anisotropy, has also been suggested (Ishii \& Dziewoński 2002; Beghein \& Trampert 2003; Cormier \& Stroujkova 2005; Niu \& Chen 2008; Sun \& Song $2008 \mathrm{a}, \mathrm{b})$, although the data poorly constrain this region of the core (Calvet et al. 2006). The observation of a strong coda following $P K i K P$ waves provides evidence that texture heterogeneities are present down to kilometre scale, at least in the upper $300 \mathrm{~km}$ of the 
inner core (Vidale \& Earle 2000; Poupinet \& Kennett 2004; Peng et al. 2008).

From a geodynamic point of view, this complexity is intriguing. A wealth of models have been proposed during the last two decades, most of them relying on the preferential alignment of anisotropic iron crystals to produce the elastic anisotropy. Lattice preferred orientation might have been either frozen-in at the inner core boundary (ICB) during solidification (Karato 1993; Bergman 1997; Brito et al. 2002), or might have developed within the inner core as a result of plastic deformation (Jeanloz \& Wenk 1988; Karato 1999; Wenk et al. 2000; Buffett \& Wenk 2001) or stressinduced recrystallization (Yoshida et al. 1996). Post-solidification mechanisms include thermal convection (Jeanloz \& Wenk 1988; Weber \& Machetel 1992; Wenk et al. 2000), viscous relaxation of an inner core topography induced by heterogeneous crystallization (Yoshida et al. 1996), and flow induced by the core magnetic field (Karato 1999; Buffett \& Wenk 2001; Takehiro 2010).

Most of the post-solidification mechanisms described above rely on predominantly radial flow, and their viability depends on whether the density profile in the inner core is stably stratified or not. A stable stratification off course precludes thermal convection, but is also more generally expected to inhibit vertical motion (Buffett \& Bloxham 2000; Deguen \& Cardin 2009). If the inner core is unstably stratified, convection can develop, and may couple with the magnetic field (Karato 1999; Buffett \& Wenk 2001) or with ICB topography relaxation (Yoshida et al. 1996). It has been recently proposed that thermal convection can take the form of a 'convective translation' of the inner core with melting in one hemisphere and solidification in the other (Monnereau et al. 2010; Alboussière et al. 2010) and, again, this requires the inner core to be unstably stratified. Whether the inner core is stably stratified or not is a first-order discriminating factor for candidate anisotropy-producing mechanisms. It is, in that sense, probably the most important unanswered question regarding inner core dynamics.

The thermal state of the inner core has been a long-standing issue. Thermal convection (Jeanloz \& Wenk 1988) has been the first mechanism proposed to explain the inner core seismic anisotropy, then recently discovered (Poupinet et al. 1983; Morelli et al. 1986; Woodhouse et al. 1986). Radiogenic heating has been initially proposed as the chief energy source for inner core convection, but the most effective source of convective instability in fact appears to be secular cooling [Yukutake (1998); and see Section 2]. The inner core may develop an unstable temperature profile simply because the solidification temperature of the core mixture is a decreasing function of radius: the temperature at the ICB decreases as the inner core grows, so the inner core is effectively cooled from above, a possibly unstable configuration. The temperature profile in the inner core results from a competition between extraction of the inner core internal heat by diffusion, and cooling at the ICB, which is controlled by the core thermal history and, ultimately, by the heat flux extracted at the core-mantle boundary (CMB). Thermal convection further requires the cooling rate of the ICB to be large enough to sustain a superadiabatic geotherm within the inner core. This is not an easy condition to fulfil because thermal conduction alone evacuates a large part of the inner core internal heat on a timescale similar to the age of the inner core (Stacey 1995; Yukutake 1998). For this reason, thermal convection in the inner core has often been thought implausible (Yukutake 1998). Yet, the low value of inner core thermal conductivity recently proposed (Stacey \& Davis 2008) and the relatively large CMB heat flux currently favoured (Lay et al. 2008) both argue in its favour and warrants a reevaluation of the possibility of inner core thermal convection (Buffett 2009).
The problem of inner core convection is further complicated by the possible presence of a chemical stratification, which may arises as a result of solidification-induced partitioning of the light elements present in the core (Stacey 1995; Deguen \& Cardin 2009). Since chemically and thermally induced density variations can be of the same order of magnitude, the interplay between thermal and compositional fields may be of significant importance for the inner core dynamics.

In this paper, we first present an analytical model of the thermal evolution of the inner core, which couples explicitly the inner core thermal state to the outer core thermal evolution (Section 2). The analysis is more general than those previously published (Sumita et al. 1995; Yukutake 1998; Buffett 2000, 2009; Deguen \& Cardin 2009), and allows us to elucidate the relative importance of the various factors involved and to assess the uncertainties involved. We then discuss the chemical state of the inner core and the possible presence of chemical stratification (Section 3). The details of a model of thermochemical convection in a growing inner core are presented in Section 4, and numerical simulations of thermochemical convection in the inner core are presented and discussed in Section 5.

\section{THERMAL EVOLUTION OF THE INNER CORE}

\subsection{Thermal evolution with no radiogenic heating}

A necessary condition for thermal convection in the inner core is that the temperature gradient is larger than the adiabatic gradient, given by

$\frac{\partial T_{\mathrm{ad}}}{\partial r}=-\frac{\rho g \gamma T}{K_{S}}=-\frac{\rho g^{\prime} \gamma T}{K_{S}} r$

where $T$ is the temperature, $\rho$ is the density, $r$ the radius, $g$ is the acceleration of gravity, $g^{\prime}=d g / d r, \gamma$ the Gruneisen parameter and $K_{S}$ the isentropic bulk modulus. In the inner core, $g$ increases almost linearly with radius (Dziewonski \& Anderson 1981), and $g^{\prime}$ will be assumed to be constant.

This suggests the use of a potential temperature, defined as

$\Theta(\mathbf{r}, t)=T-T_{\text {ad }}(r, t)$,

where the adiabat $T_{\mathrm{ad}}(r, t)$ is anchored at the ICB [i.e. $\Theta\left(r_{\mathrm{ic}}, t\right)=0$, with $r_{\text {ic }}$ the radius of the inner core]. The inner core is superadiabatic, and may convect, if $\Theta$ increases with depth, and is subadiabatic otherwise. Following standard practice in atmospheric science (see Tritton 1988), we can take advantage of the fact that the Dissipation number,

$D i=\frac{\alpha g r_{\mathrm{ic}}}{c_{p}}=\frac{\rho g \gamma r_{\mathrm{ic}}}{K_{S}} \simeq 0.06 \times\left(\frac{r_{\mathrm{ic}}}{1221 \mathrm{~km}}\right)^{2}$,

is small compared to one, to simplify the equation of conservation of entropy to

$\frac{D \Theta}{D t}=\kappa \nabla^{2} \Theta+S(t)$,

where $\kappa$ is the thermal diffusivity in the inner core, $\alpha$ the thermal expansivity and $c_{p}$ the specific heat. $S(t)$ is defined as

$$
\begin{aligned}
S(t) & =\kappa \nabla^{2} T_{\mathrm{ad}}-\frac{\partial T_{\mathrm{ad}}}{\partial t} \\
& =-3 \kappa \frac{\rho g^{\prime} \gamma T}{K_{S}}+\left(\left.\frac{\partial T_{\mathrm{ad}}}{\partial r}\right|_{\mathrm{icb}}-\left.\frac{\partial T_{\mathrm{s}}}{\partial r}\right|_{\mathrm{icb}}\right) u_{\mathrm{ic}}
\end{aligned}
$$


$T$

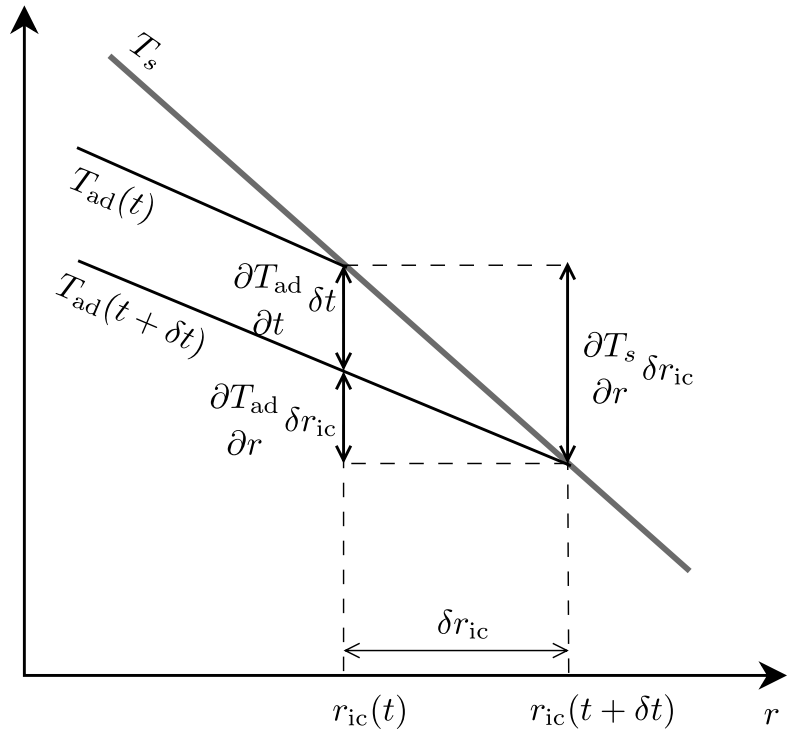

Figure 1. Cooling of the inner core adiabat anchored at the ICB. The ICB is at radius $r_{\mathrm{ic}}(t)$ at time $t$, and grows to $r_{\mathrm{ic}}(t+\delta t)=r_{\mathrm{ic}}(t)+\delta r_{\mathrm{ic}}$ during the time interval $\delta t$. Meanwhile, the adiabat anchored at the ICB, initially $T_{\text {ad }}(t)$, drops to $T_{\text {ad }}(t+\delta t)$. As shown in the figure, the adiabat drop during $\delta t,\left(\partial T_{\text {ad }} / \partial t\right) \delta t$, is equal to $\left(\partial T_{\mathrm{s}} / \partial r\right) \delta r_{\text {ic }}-\left(\partial T_{\text {ad }} / \partial r\right) \delta r_{\text {ic }}$, which implies that $\left(\partial T_{\mathrm{ad}} / \partial t\right)=\left[\left(\partial T_{\mathrm{s}} / \partial r\right)_{\mathrm{icb}}-\left(\partial T_{\mathrm{ad}} / \partial r\right)_{\mathrm{icb}}\right] u_{\mathrm{ic}}$, the solidification rate $u_{\mathrm{ic}}$ being equal to $\delta r_{\mathrm{ic}} / \delta t$.

where $T_{\mathrm{s}}$ is the solidification temperature of the core mixture and $u_{\text {ic }}$ the inner core solidification rate (see Fig. 1 for the calculation of $\left.\partial T_{\mathrm{ad}} / \partial t\right) . S(t)$ is the sum of a constant sink term associated with conduction along the adiabat and of a source term due to cooling at the ICB (more precisely the rate of cooling of the adiabat anchored at the ICB). It is clear from this formulation that cooling at the ICB is mathematically equivalent to internal heating at a rate imposed by the cooling rate of the ICB. Sustained thermal convection requires $S(t)$ to be positive ${ }^{1}$, which requires that the cooling rate of the inner core is too large to be accommodated by conduction along the adiabat.

Noting that

$\left.\frac{\partial T_{\mathrm{ad}}}{\partial r}\right|_{\mathrm{icb}}=-\frac{\rho g^{\prime} \gamma T}{K_{S}} r_{\mathrm{ic}}(t)$

and neglecting the radial variations of temperature in the expression of the adiabatic gradient, eq. (6) can be rewritten as

$S(t)=\frac{\rho g^{\prime} \gamma T}{K_{S}}\left[\left(\frac{d T_{\mathrm{s}}}{d T_{\mathrm{ad}}}-1\right) r_{\mathrm{ic}}(t) u_{\mathrm{ic}}(t)-3 \kappa\right]$,

where $d T_{\mathrm{s}} / d T_{\text {ad }}$ is the ratio of the Clapeyron slope to the adiabat. $d T_{\mathrm{s}} / d T_{\text {ad }}$ will be assumed constant in the pressure range of the inner core.

The requirement that $S$ must be positive for thermal convection to occur gives a necessary criterion for thermal convection, namely that

$\frac{d r_{\mathrm{ic}}^{2}}{d t}>\frac{6 \kappa}{\frac{d T_{\mathrm{s}}}{d T_{\mathrm{ad}}}-1}$.

\footnotetext{
${ }^{1}$ This is in general not a necessary condition for convection: transient convection may still occur even if $S<0$ in a configuration where the initial temperature profile is superadiabatic. In practice however, the inner core, having been growing from zero, does not have any initial internal heat, and the initiation of convection requires that $S>0$.
}

It is instructive to momentarily simplify the problem further and assume that the inner core grows as the square root of time. With $r_{\text {ic }} \propto \sqrt{t}$, then $d\left(r_{\text {ic }}^{2}\right) / d t$ is constant and equals to $r_{\text {ic }}^{* 2} / \tau_{\text {ic }}$, where $r_{\text {ic }}^{*}$ is the present radius of the inner core and $\tau_{\text {ic }}$ the age of the inner core. $S$ does not depend on time and the criterion (9) can be written as a criterion for the age of the inner core (Deguen \& Cardin 2009): thermal convection is possible if

$\tau_{\text {ic }}<\tau_{\kappa}\left(\frac{d T_{\mathrm{s}}}{d T_{\mathrm{ad}}}-1\right)$,

where $\tau_{\kappa}=r_{\mathrm{ic}}^{* 2} /(6 \kappa)$ is the present thermal diffusion time in the inner core. This gives a first-order estimate of the age of the inner core needed for thermal convection, and has the advantage of making apparent the sensitivity of the thermal stratification limit to the relevant parameters. The occurrence of thermal convection depends directly on the thermal diffusion timescale, but is also highly sensitive to the ratio of the Clapeyron and adiabat slopes, which is a measure of the maximum internal heat available for convection. Eq. (10) also suggests that, even with a more realistic core thermal history, whether the inner core is superadiabatic or not depends at first order on the parameter

$\mathcal{T}_{\text {ic }}=\left(\frac{d T_{\mathrm{s}}}{d T_{\mathrm{ad}}}-1\right)^{-1} \frac{\tau_{\mathrm{ic}}}{\tau_{\kappa}}$.

Uncertainties on $\tau_{\kappa}$ are mainly due to uncertainties on the thermal conductivity of iron at inner core conditions. The relatively large value $\left(k=79 \mathrm{~W} \mathrm{~m}^{-1} \mathrm{~K}^{-1}\right)$ favoured by Stacey \& Anderson (2001) has been recently revised downward, with Stacey \& Davis (2008) giving $k=36 \mathrm{~W} \mathrm{~m}^{-1} \mathrm{~K}^{-1}$.

The ratio $d T_{\mathrm{s}} / d T_{\mathrm{ad}}$ can be estimated by using Lindeman's law,

$\frac{d T_{\mathrm{s}}}{d P}=\frac{2(\gamma-1 / 3) T}{K_{T}}$,

and writing the adiabatic temperature gradient as

$\frac{d T_{\mathrm{ad}}}{d P}=\frac{\gamma T}{K_{S}}$,

where $P$ is the pressure and $K_{T}$ the isothermal bulk modulus. Using the thermodynamic identity $K_{S}=K_{T}(1+\gamma \alpha T)$, we find

$\frac{d T_{\mathrm{s}}}{d T_{\mathrm{ad}}}=\frac{2(\gamma-1 / 3)(1+\gamma \alpha T)}{\gamma}$.

With the values and uncertainties of thermophysical properties given in Table 1, we find $\tau_{\kappa}=1.4 \pm 0.7 \mathrm{~Gy}$, and $d T_{\mathrm{s}} / d T_{\mathrm{ad}}=1.65 \pm$ 0.11 . This gives a critical age for superadiabaticity of $0.9 \pm 0.6 \mathrm{~Gy}$. The range of critical ages we obtain fully overlaps with what models of core thermal evolution predict for the age of the inner core, for example, $1 \pm 0.5 \mathrm{~Gy}$ in Labrosse et al. (2001) and $1.15 \pm 0.75 \mathrm{~Gy}$ in Nimmo (2007); the uncertainties are such that it is not currently possible to conclude about the likelihood of inner core convection.

Going back to a more general core thermal history, it is clear from eq. (8) that the evolution of $S(t)$ is governed by the core thermal history, and we therefore use the core energy balance to express $S$ as a function of time. After the nucleation of the inner core, the heat flux $Q_{\mathrm{cmb}}$ extracted at the CMB is balanced by the energy released by the secular cooling of the core, and the latent heat and compositional energy released by inner core crystallization (we ignore at this stage the possibility of radiogenic heating, and neglect smaller contributions from other energy sources). The core energy balance can be written as

$Q_{\mathrm{cmb}}=\left[P_{c}\left(r_{\mathrm{ic}}\right)+P_{L}\left(r_{\mathrm{ic}}\right)+P_{G}\left(r_{\mathrm{ic}}\right)\right] \frac{d r_{\mathrm{ic}}}{d t}$, 
Table 1. Thermophysical parameters.

\begin{tabular}{|c|c|c|}
\hline Parameter & Symbol & Value \\
\hline Inner core radius $^{a}$ & $r_{\text {ic }}$ & $1221 \mathrm{~km}$ \\
\hline Core radius $^{a}$ & $r_{\mathrm{c}}$ & $3480 \mathrm{~km}$ \\
\hline Solidification temperature ${ }^{b}$ & $T_{\text {icb }}$ & $5600 \pm 500 \mathrm{~K}$ \\
\hline Gruneisen parameter $^{c}$ & $\gamma$ & $1.4 \pm 0.1$ \\
\hline Thermal expansivity $^{c}$ & $\alpha$ & $(1.1 \pm 0.1) \times 10^{-5} \mathrm{~K}^{-}$ \\
\hline Heat capacity ${ }^{d}$ & $c_{p}$ & $800 \pm 80 \mathrm{~J} \mathrm{~kg}^{-1} \mathrm{~K}^{-1}$ \\
\hline Density in the inner core ${ }^{a}$ & $\rho$ & $13000 \mathrm{~kg} \mathrm{~m}^{-3}$ \\
\hline Thermal conductivity ${ }^{e}$ & $k$ & $36-79 \mathrm{~W} \mathrm{~m}^{-1} \mathrm{~K}^{-1}$ \\
\hline Isentropic bulk modulus $^{a}$ & $K_{S}$ & $1.3 \mathrm{GPa}$ \\
\hline $\begin{array}{l}\text { Thermal diffusion timescale } \\
\text { Clapeyron/adiabat ratio }\end{array}$ & $\begin{array}{l}\tau_{\kappa} \\
d T_{\mathrm{S}} / d T_{\mathrm{ad}}\end{array}$ & $\begin{array}{l}1.4 \pm 0.7 \mathrm{~Gy} \\
1.65 \pm 0.11\end{array}$ \\
\hline Initial concentration ${ }^{b}$ & $c_{0}$ & $5.6 \mathrm{wt} \%$ \\
\hline Partition coefficient ${ }^{b}$ & $D$ & 0.8 \\
\hline Chemical expansivity $^{f}$ & $\alpha_{c}$ & -1 \\
\hline \multicolumn{3}{|c|}{$\begin{array}{l}{ }^{a} \text { From PREM (Dziewonski \& Anderson 1981). } \\
b \text { Alfè et al. (2002). } \\
{ }^{c} \text { Vočadlo (2007). } \\
d \text { Poirier (1994); Stacey \& Davis (2008). } \\
{ }^{e} \text { Stacey \& Anderson (2001); Stacey \& Davis (2008). } \\
{ }^{\circ} \text { See text. }\end{array}$} \\
\hline
\end{tabular}

where $P_{c}, P_{L}$ and $P_{G}$ are functions of the inner core radius which accounts for the relative contributions of respectively secular cooling, latent heat and compositional energy. We use here the formulation of Labrosse (2003), to which the reader is invited to refer to for the details of the model. Other formulations of the core energy balance (e.g. Buffett et al. 1996; Gubbins et al. 2004) only differ in the parametrization of the core state, and would give similar results. Integrating eq. (15) since the onset of inner core crystallization gives the age of the inner core as $\tau_{\mathrm{ic}}=E_{\mathrm{tot}} / \bar{Q}_{\mathrm{cmb}}$, where $E_{\mathrm{tot}}=\int_{\mathrm{ic} 0}^{r *}\left(P_{c}+\right.$ $\left.P_{L}+P_{G}\right) d r_{\text {ic }}$ is the total energy which must be extracted from the core to crystallize the inner core to its present size, and $\bar{Q}_{\mathrm{cmb}}$ is the mean CMB heat flow over the life of the inner core. We assume here a constant $Q_{\mathrm{cmb}}$ - a time-dependent heat flux is straightforward to include, but the secular evolution of $Q_{\mathrm{cmb}}$ is uncertain - and rewrite eq. (15) as

$r_{\mathrm{ic}} u_{\mathrm{ic}}=\frac{Q_{\mathrm{cmb}} r_{\mathrm{ic}}}{P_{c}+P_{L}+P_{G}}=\frac{E_{\mathrm{tot}}}{P_{c}+P_{L}+P_{G}} \frac{r_{\mathrm{ic}}}{\tau_{\mathrm{ic}}}$,

which is inserted in eq. (8) to give

$$
\begin{aligned}
S(t) & =\frac{\rho g^{\prime} \gamma T}{K_{S}} 3 \kappa\left[f\left(r_{\mathrm{ic}}\right)\left(\frac{d T_{\mathrm{s}}}{d T_{\mathrm{ad}}}-1\right) \frac{\tau_{\kappa}}{\tau_{\mathrm{ic}}}-1\right], \\
& =\frac{\rho g^{\prime} \gamma T}{K_{S}} 3 \kappa\left[f\left(r_{\mathrm{ic}}\right) \mathcal{T}_{\mathrm{ic}}^{-1}-1\right],
\end{aligned}
$$

where

$$
f\left(r_{\mathrm{ic}}\right)=2 \frac{E_{\mathrm{tot}}}{\left(P_{c}+P_{L}+P_{G}\right) r_{\mathrm{ic}}^{*}} \frac{r_{\mathrm{ic}}}{r_{\mathrm{ic}}^{*}}
$$

is an $\mathcal{O}(1)$ function of $r_{\text {ic }}$. The expression of $S(t)$ could have been written equivalently as a function of $Q_{\mathrm{cmb}}$ since $Q_{\mathrm{cmb}} \tau_{\mathrm{ic}}=E_{\mathrm{tot}}$, but the results are by far less sensitive to uncertainties in core properties when expressed in term of $\tau_{\text {ic }}$. It is important to realize that there are very large uncertainties on the value of $E_{\text {tot }}$-Labrosse (2003) gives $E_{\mathrm{tot}}=(29.3 \pm 18.8) \times 10^{28} \mathrm{~J}$. When $S$ is written as a function of $\tau_{\text {ic }}$, all uncertainties related to the core thermal model appear in $f$ as the ratio of $E_{\text {tot }}$ to $P_{c}+P_{L}+P_{G}$, whose magnitude is insensitive to the core thermal model uncertainties.

Fig. 2 shows the evolution of $S$ during the growth of the inner core, for $k=36 \mathrm{~W} \mathrm{~m}^{-1} \mathrm{~K}^{-1}$ and $k=79 \mathrm{~W} \mathrm{~m}^{-1} \mathrm{~K}^{-1}$, and various

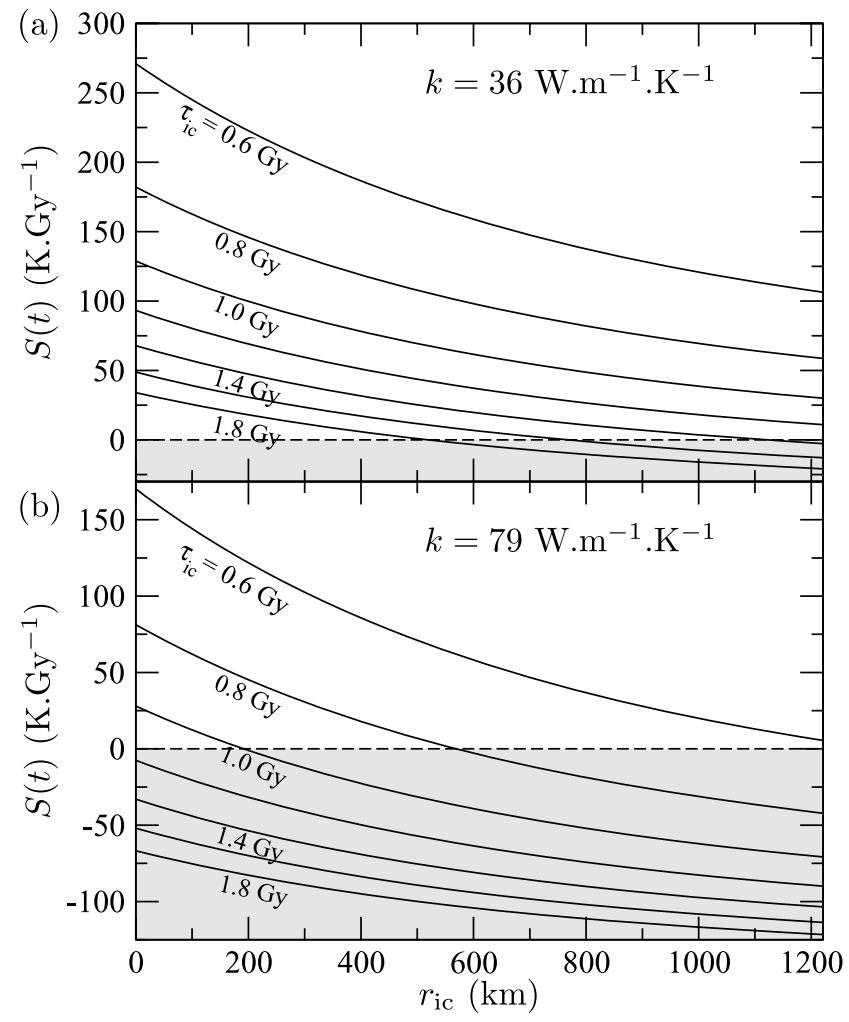

Figure 2. Value of $S$ as a function of inner core radius $r_{\text {ic }}$, for various values of age of the inner core $\tau_{\text {ic }}$. (a) With $k=36 \mathrm{~W} \mathrm{~m}^{-1} \mathrm{~K}^{-1}$; (b) With $k=$ $79 \mathrm{~W} \mathrm{~m}^{-1} \mathrm{~K}^{-1}$. Calculations were made using the core thermal evolution model of Labrosse (2003).

values of $\tau_{\text {ic }}$ (values of the other parameters involved are given in Table 1). $S(t)$ is a decreasing function of time because the cooling of the core and the growth of the inner core are gradually slowed down by the release of latent heat and gravitational energy associated with crystallization. This implies that the inner core is more likely to be superadiabatic in its early history. This tendency would be accentuated if $Q_{\mathrm{cmb}}$ is assumed to decrease with time. The range of values of $\tau_{\text {ic }}$ used in Fig. 2 is representative of the uncertainty on the age of the inner core arising from uncertainties on both $Q_{\mathrm{cmb}}$ and on the thermophysical properties of the core. Thermal convection thus appears likely if $k=36 \mathrm{~W} \mathrm{~m}^{-1} \mathrm{~K}^{-1}$, but quite unlikely if $k$ is as large as $79 \mathrm{~W} \mathrm{~m}^{-1} \mathrm{~K}^{-1}$. Note that $S$ can be quite large; for comparison, the heating rate in the mantle by radioactive decay is of the order of $100 \mathrm{~K} \mathrm{~Gy}^{-1}$ (assuming a total heat production of 20 TW in the mantle).

Fig. 3 summarizes the results on the thermal state of the inner core in the form of regime diagrams. Fig. 3(a) shows the limit between the stable (subadiabatic) and unstable (superadiabatic) fields, as a function of $\mathcal{T}_{\text {ic }}$ and $r_{\text {ic }}(t)$. From eq. (18), the limit between the superadiabatic and subadiabatic fields is simply given by $\mathcal{T}_{\text {ic }}=f\left(r_{\text {ic }}\right)$. The grey shading gives the uncertainties on this limit arising from uncertainties in the core thermophysical parameters. The evolution of $f$ with $r_{\text {ic }}(t)$ depends on the relative importance of secular cooling, latent heat and compositional energy in the core energy budget, but its average magnitude is insensitive to the core thermal model. This demonstrates that the thermal state of the inner core is mostly determined by the value of the non-dimensional inner core age $\mathcal{T}_{\text {ic }}$, and that the simplified criterion given in eq. (10) indeed gives a good estimate of the conditions needed for thermal convection. Fig. 3(b) is a dimensional version of Fig. 3(a), and shows the stability fields 


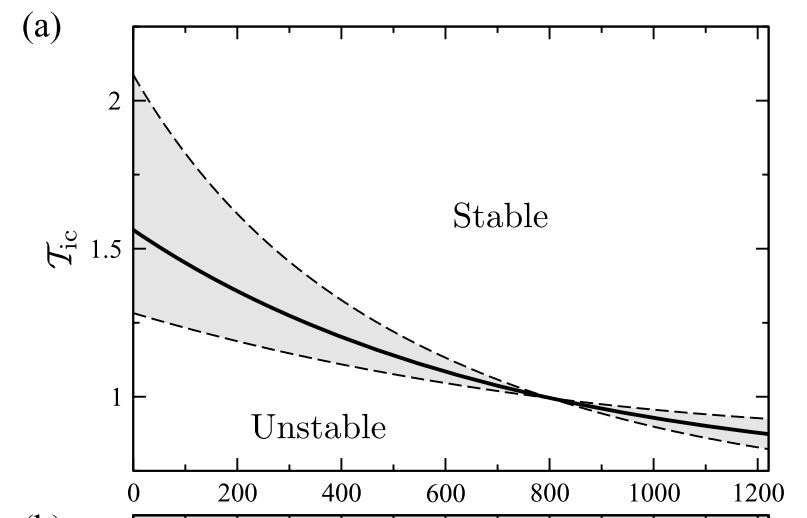

(b)

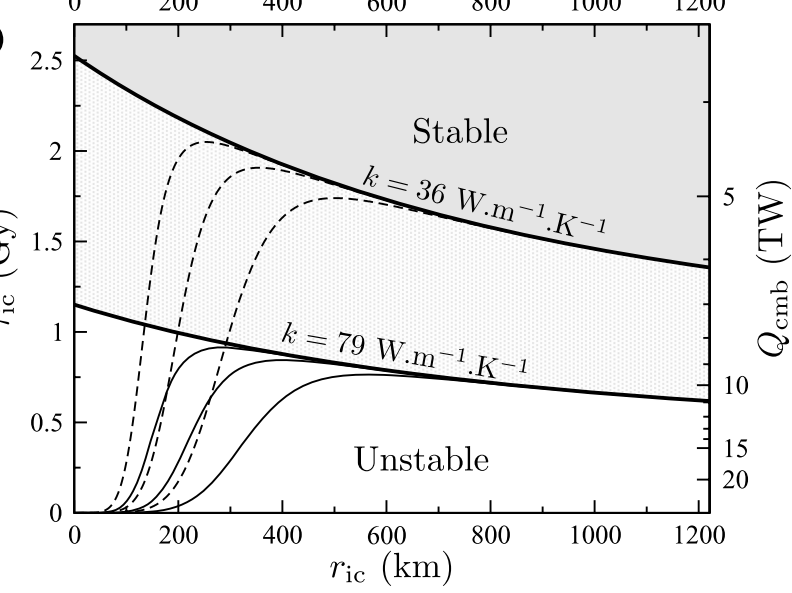

Figure 3. (a) Stability diagram of the inner core as a function of $\mathcal{T}_{\text {ic }}$ [eq. (11)] and radius of the inner core at time $t, r_{\mathrm{ic}}(t)$. The grey shading shows the uncertainty on the location of the stability limit. (b) Stability diagram of the inner core as a function of the age of the inner core (or given a core model, heat flux at the $\mathrm{CMB}$ ) and $r_{\mathrm{ic}}(t)$. Each labelled curve separates the domain where the inner core is superadiabatic (on the right upper side) from the domain where it is subadiabatic (left lower side), for thermal conductivity $k=36$ and $79 \mathrm{~W} \mathrm{~m}^{-1} \mathrm{~K}^{-1}$. Thin solid lines: radius of the inner core at which the Rayleigh number equals the critical value for thermal convection, calculated with a dynamic viscosity $\eta$ equal to $10^{17}, 10^{18}$ and $10^{19} \mathrm{~Pa} \mathrm{~s}$ (from left hand side to right hand side) with $k=79 \mathrm{~W} \mathrm{~m}^{-1} \mathrm{~K}^{-1}$; thin dashed lines: the same with $k=36 \mathrm{~W} \mathrm{~m}^{-1} \mathrm{~K}^{-1}$.

as a function of the age of the inner core and $r_{\text {ic }}$, calculated with the core parameters given in Table 1 and different values of the thermal conductivity. For illustration, we also give the value of $Q_{\mathrm{cmb}}$ corresponding to $\tau_{\text {ic }}$, calculated with $E_{\text {tot }}$ taken to be $29.1 \times 10^{28} \mathrm{~J}$.

Convection further requires that the Rayleigh number (based on the potential temperature difference) exceeds a critical value, but this appears to be a somewhat secondary issue. The large length scales involved by itself ensure that the Rayleigh number is supercritical provided the geotherm is only slightly superadiabatic. The thin dashed and solid curves in Fig. 3 give the radius $r_{\mathrm{ic}}(t)$ at which the Rayleigh number of the inner core is equal to the critical Rayleigh number for infinite Prandtl number thermal convection in a self-gravitating sphere with shear stress free boundary conditions $\left[R a_{c}=3091\right.$ (Chandrasekhar 1961)], for a viscosity $\eta$ equal to $10^{17}$, $10^{18}$ and $10^{19} \mathrm{~Pa} \mathrm{~s}$.

\subsection{The effect of radiogenic heating}

Radiogenic heating has been initially proposed as the chief energy source for inner core thermal convection (Jeanloz \& Wenk 1988; Weber \& Machetel 1992; Wenk et al. 2000). Yet, its effect is not as intuitive as it may seem at first view, and it is in fact not even clear that it will help convection. The reason why is that the presence of radiogenic heating in the core contributes to the core global heat balance and results in a more gradual inner core growth. The slower inner core growth allows more time to evacuate the inner core internal heat by conduction, hence counteracting the effect of the additional radiogenic heat source within the inner core.

Assuming non negligible radiogenic heating, the core global energy balance now writes

$Q_{\mathrm{cmb}}=\left[P_{c}+P_{L}+P_{G}\right] \frac{d r_{\mathrm{ic}}}{d t}+Q_{r}$,

where $Q_{r}=(4 / 3) \pi r_{c}^{3} \bar{\rho} \bar{h}$ is the total radiogenic heating in the core, $r_{c}$ is the radius of the core, and $\bar{h}$ is the mean radiogenic heating per unit mass in the core. $\bar{h}$ evolves with time as

$\bar{h}(t)=\bar{h}_{0} \mathrm{e}^{-\lambda t}$,

where $\bar{h}_{0}=1.917 \times 10^{-9} \times c_{K} \mathrm{~W} \mathrm{~kg}^{-1}$ is the present heat production for a potassium massic concentration $c_{K}$, and $\lambda=0.5544 \mathrm{~Gy}^{-1}$ is the decay constant. For a given CMB heat flux, taking into account radiogenic heating in the core would add a term in eq. (4) equal to

$\Delta S_{r}(t)=\frac{h_{\mathrm{ic}}}{c_{p}}-\frac{\alpha g^{\prime} \bar{T}}{c_{p}}\left(\frac{d T_{\mathrm{s}}}{d T_{\mathrm{ad}}}-1\right) \frac{r_{\mathrm{ic}}}{P_{c}+P_{L}+P_{G}} Q_{r}$,

where $h_{\text {ic }}$ is the rate of radiogenic heating per unit mass in the inner core. The first term corresponds to radiogenic heating within the inner core, and the second term, which is negative, arise because of the decrease in magnitude of the secular cooling. The relative importance of these two effects depends on the partitioning behaviour of potassium during solidification, so we write $h_{\text {ic }}$ as a fraction $D_{K}$ of the radiogenic heat production $h_{\mathrm{oc}}$ in the outer core. Assuming $h_{\mathrm{oc}} \simeq \bar{h}$, which is a good approximation if $D_{K}$ is not too large, we write

$\Delta S_{r}(t)=\frac{\bar{h}(t)}{c_{p}}\left[D_{K}-\frac{4 \pi}{3}\left(\frac{d T_{\mathrm{s}}}{d T_{\mathrm{ad}}}-1\right) \frac{\alpha g^{\prime} \bar{T} \bar{\rho} r_{c}^{3} r_{\mathrm{ic}}}{P_{c}+P_{L}+P_{G}}\right]$.

The second term in the parentheses is of order 1 during all inner core history: radiogenic heating in the core will make inner core convection more difficult if the outer core is enriched in radioactive elements compared to the inner core. Both the abundance of potassium in the core and the value of $D_{K}$ are unknown. Experimental and theoretical studies suggest that a maximum of a few $100 \mathrm{ppm}$ of potassium may have alloyed with iron during core formation (Parker et al. 1996; Gessmann \& Wood 2002; Murthy et al. 2003; Bouhifd et al. 2007), and possibly no more than a few tens of ppm (Corgne et al. 2007); for illustration, we assume here a nominal value of $100 \mathrm{ppm}$. We plotted on Fig. 4 the evolution of $\Delta S_{r}$ during the growth of the inner core for several values of $D_{K}$ and an assumed potassium abundance of $100 \mathrm{ppm}$. Several studies suggest that potassium may readily alloy with crystalline iron (Bukowinski 1976; Lee \& Jeanloz 2003), so we consider the $D_{K}>1$ case as possible. Radiogenic heating has a small effect if $D_{K} \sim 1$, and would even have a stabilizing effect on inner core convection if potassium is incompatible $\left(D_{K}<1\right)$.

\section{COMPOSITIONAL STRATIFICATION}

Several studies have pointed out that solidification-induced partitioning of the various solutes present in the core may produce a stabilizing chemical stratificati on (Stacey 1995; Buffett 2000; Buffett \& Bloxham 2000; Buffett 2009; Deguen \& Cardin 2009). Light 


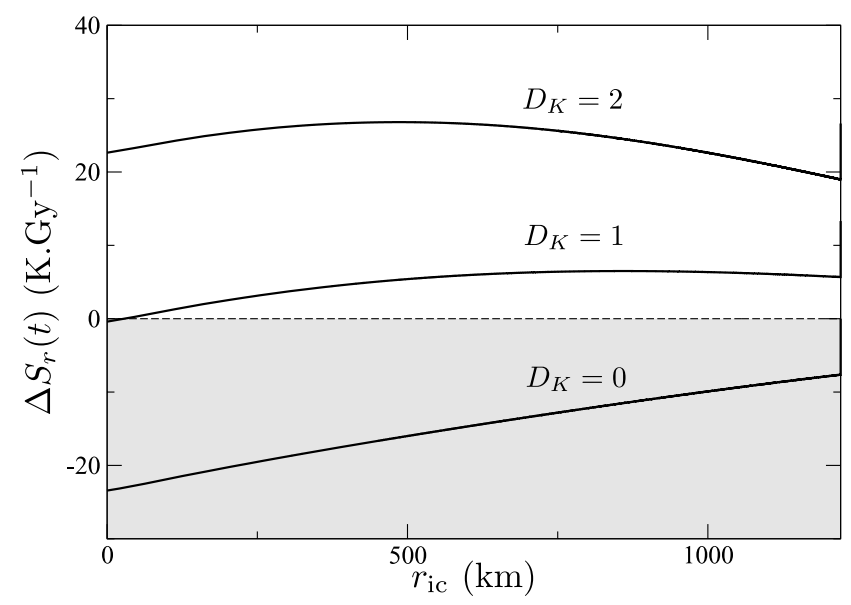

Figure 4. Value of $\Delta S_{r}$ as a function of inner core radius $r_{\text {ic }}$, for various values of the partition coefficient of potassium. Calculation were made with a core potassium concentration of $100 \mathrm{ppm}$ and an inner core age of $1 \mathrm{~Gy}$.

elements $(\mathrm{O}, \mathrm{Si}, \mathrm{S}, \ldots)$ in particular are likely to be rejected preferentially in the liquid phase when crystallization occur, and the growth of the inner core is thus expected to result in a gradual enrichment of the outer core in light elements. Since the composition of the newly crystallized solid $c^{s}$ is related to the liquid composition $c^{l}$ by the partition (or distribution) coefficient $D$ as

$c^{s}=D c^{l}$,

the chemical evolution of the outer core is expected to be imprinted in the inner core.

In all previous studies, the resulting radial chemical stratification has been calculated with the assumption of a constant distribution coefficient. While this may seem a most reasonable approximation given the lack of constraints we have on the partitioning behaviour of the core light elements, this may be an oversimplification. As pointed out by Alboussière et al. (2010), variations of $D$, while strictly unknown, can be of importance. Logarithmic differentiation of (24) gives

$\frac{d c^{s}}{c^{s}}=\frac{d c^{l}}{c^{l}}+\frac{d D}{D}$

which shows that variations of $D$ should be taken into account if the relative evolution of $D$ is comparable in magnitude to the relative compositional evolution of the outer core.

The first term on the right-hand side of eq. (25) is positive for incompatible elements and reflects the gradual enrichment of the outer core. It is given by the differential form of the Rayleigh distillation equation as

$\frac{d c^{l}}{c^{l}}=(D-1) \frac{d M_{\mathrm{oc}}}{M_{\mathrm{oc}}}=(1-D) \frac{d M_{\mathrm{ic}}}{M_{\mathrm{oc}}}$,

where $M_{\mathrm{oc}}$ and $M_{\mathrm{ic}}$ are the mass of the outer core and inner core. Noting $c_{0}^{l}$ the initial light element concentration in the liquid core, the relative chemical enrichment of the outer core since inner core nucleation, $\left(c^{l}-c_{0}^{l}\right) / c_{0}^{l}$, is of the order of $(1-D)\left(M_{\mathrm{ic}} / M_{\mathrm{oc}}\right)$, which is smaller than $M_{\mathrm{ic}} / M_{\mathrm{oc}} \simeq 5$ per cent for an incompatible element.

The second term on the right-hand side of eq. (25) is unknown, but, as pointed out by Alboussière et al. (2010), may be negative and of comparable magnitude. The partition coefficient must be thought of as an effective partition coefficient, which in practice often differs from the thermodynamic partition coefficient given by the equilibrium phase diagram (Chalmers 1964). In particular if the solidification regime of the inner core is dendritic (Fearn et al. 1981; Loper 1983; Bergman 1997; Shimizu et al. 2005; Deguen et al. 2007), then the effective partition coefficient would depend on the fraction of liquid trapped in the mushy layer (Alboussière et al. 2010). This depends on the efficiency of interdendritic convection (Loper 1983; Worster 1991) and compaction of the solid matrix (Sumita et al. 1996). Both processes being gravity driven, the efficiency of melt extraction may be expected to increase during the growth of the inner core. This will result in a decrease of the effective partition coefficient. As pointed out by Alboussière et al. (2010), relative variations of a few percent in the value of $D$ would be enough to counterbalance the effect of the secular evolution of the core chemical composition.

Note that change with pressure of the thermodynamic distribution coefficient can in theory lead to a similar effect. It is known-and has been observed, for example, for some trace elements in iron meteorites (e.g. Chabot \& Drake 1999) - that the concentration in incompatible elements in the solid phase may actually decrease in the course of solidification if the partition coefficient decreases (the decrease in $D$ being here due to the evolution of the liquid phase composition).

Since variations of $D$ are unknown, we will assume here that $D$ remains constant and treat this case as an end-member case, bearing in mind that the magnitude of the stratification we calculate may be overestimated. With this assumption, the compositional profile in the inner core is given by combining the Rayleigh distillation equation (eq. 26) with the definition of $D$. Neglecting radial density variations in the core, the light element concentration $c^{s}$ in the inner core is given as

$c^{s}=D c_{0}^{l}\left[1+\left(\frac{r}{r_{\mathrm{c}}}\right)^{3}\right]^{1-D}$

(Deguen \& Cardin 2009). Taking into account compressibility (radial density variations in the core) and the density jump at the ICB would result in a stratification approximately 15 per cent larger. The chemically induced density difference between the ICB and the centre of the inner core is

$\Delta \rho_{c}=\alpha_{c} \rho\left[c^{s}\left(r_{\mathrm{ic}}\right)-c^{s}(0)\right] \sim \alpha_{c} \rho c_{0}^{l} D(1-D)\left(\frac{r}{r_{\mathrm{c}}}\right)^{3}$,

where $\alpha_{c}=(1 / \rho)(\partial \rho) /(\partial c)$ is the chemical expansion coefficient. Compositional variations within the inner core would be maximum for moderately incompatible elements with $D \sim 0.5$, and would be small for elements with either $D \ll 1$ or $D$ close to 1 .

The magnitude of the stratification depends on the nature and abundance of the light elements present in the core. The composition of the core has been a long standing issue and is still controversial, but recent models favour $\mathrm{O}, \mathrm{Si}$ and $\mathrm{S}$ as the most plausible alloying elements. Ab initio calculations of the partitioning behaviour of $\mathrm{O}$, $\mathrm{Si}$ and $\mathrm{S}$ by Alfè et al. (2002) suggest that $\mathrm{Si}$ and $\mathrm{S}$ both partition weakly (with similar partition coefficients, $D^{\mathrm{Si}, \mathrm{S}}=0.8$ ), while in contrast $\mathrm{O}$ partitions strongly, $D^{\mathrm{O}}=0.02$ (values of $D$ are converted from molar ratios to mass ratios). These values favour the presence of sulphur or silicium in the inner core. Using these distribution coefficients and the seismological estimates of the density of the core and of the density jump at the ICB, Alfè et al. (2002) estimate the composition of the outer core to be $5.6 \mathrm{wt} . \%$ of $\mathrm{Si}$ and/or S and 2.5 wt. $\%$ of $\mathrm{O}$, in good agreement with the geochemical model of Allègre et al. (1995), and 4.4 wt.\% of Si/S in the inner core and negligible amount of $\mathrm{O}$. 
We estimate in appendix the chemical expansion coefficients of light elements in the inner core, from values of partial atomic volumes of $\mathrm{Fe}, \mathrm{S}, \mathrm{Si}$ and $\mathrm{O}$ given by Alfè et al. (2002). With these estimates of $\alpha_{c}$ and the composition model of Alfè et al. (2002), we find that the density stratification associated with $\mathrm{Si} / \mathrm{S}$ is much larger than that associated with $\mathrm{O}$, and we therefore model the inner core as a $\mathrm{Fe}-(\mathrm{Si}, \mathrm{S})$ binary mixture, with a distribution coefficient equal to 0.8 . With the value listed in Table 1 , we find a present difference of density $\Delta \rho_{c} \sim-5 \mathrm{~kg} \mathrm{~m}^{-3}$ between the centre of the inner core and the ICB. Note that we have considered in a first approximation that the partition coefficient and the compositional expansion coefficient do not vary. This result also supposes perfect mixing in the outer core, thus ignoring the possible accumulation of light element rich liquid below the CMB (Fearn \& Loper 1981) or the presence of a dense layer in the lowermost liquid outer core (Souriau \& Poupinet 1991; Alboussière et al. 2010). Chemical interaction between melt in the outer core and silicate material at the $\mathrm{CMB}$, which might buffer the composition of the outer core (Buffett et al. 2000), are also assumed negligible.

\section{A NUMERICAL MODEL OF THERMOCHEMICAL CONVECTION IN A GROWING INNER CORE}

We now present and discuss the set of equations we use to model inner core thermochemical convection.

We assume here that the ICB, while being a phase change interface, can be treated as impermeable (i.e. there is no phase change allowed except the gradual crystallization of the inner core associated with secular cooling). This requires justification, and we discuss briefly this assumption in Section 4.2.

An important particularity of the present model is that we explicitly take into account the inner core growth, starting with a small inner core 'nucleus' which we let grow according to the core energy balance. The thermal forcing and chemical stratification also evolve with time, and are derived from the core thermal and chemical evolution, as explained in the two preceding sections.

\subsection{Governing equations}

The analysis presented in Section 2.2 suggests that radiogenic heating is not critical for inner core convection and we will thus ignore it in our numerical simulations to avoid yet another free parameter. Convection is considered to be driven by secular cooling alone. The evolution of the potential temperature is then governed by eq. (4). The evolution of the compositional field is given by the equation of conservation of solute,

$\frac{D c}{D t}=\kappa_{c} \nabla^{2} c$,

where $\kappa_{c}$ is the chemical diffusivity in the inner core. Variations of density with temperature and composition are given by a linearized equation of state, which is written as

$\rho=\rho_{0}\left[1-\alpha \Theta+\beta\left(c-D c_{0}^{l}\right)\right]$,

where $D c_{0}^{l}$ is the light element concentration at the centre of the inner core, and $\rho_{0}$ is a reference density.

The rheology is assumed to be Newtonian, and, in addition, the viscosity is assumed to be independent of temperature and pressure. The effect of self-gravitation originating from density perturbations can be shown to be small in the inner core, and will be ignored here. The equation of momentum conservation reduces to the Stokes equation at vanishingly small Re number, with thermal and compositional buoyancy terms, and is expressed as

$0=-\nabla p+\rho(\Theta, c) \mathbf{g}+\eta \nabla^{2} \mathbf{u}$,

where $\mathbf{g}=-g \mathbf{e}_{\mathbf{r}}$ is the gravitational acceleration, $\mathbf{e}_{\mathbf{r}}$ is the unit radial vector, and $\eta$ is the dynamic viscosity. Within the Boussinesq approximation framework, conservation of mass writes

$\nabla \cdot \mathbf{u}=0$.

\subsection{Boundary conditions}

We assume here that the ICB can be treated as an impermeable surface, assuming no phase change associated with convectioninduced radial displacement of the ICB. This has been a simplifying hypothesis of previous analysis of inner core thermal convection (Weber \& Machetel 1992; Wenk et al. 2000; Buffett 2009). In contrast, Karato (1999), 2000) and Takehiro (2010) treated the ICB as a perfectly permeable boundary, assuming that phase change is instantaneous when the solidification front is shifted radially. As emphasized by Alboussière et al. (2010), neither approach is strictly correct: solidification and melting are rate-limited by the ability of outer core convection to supply or evacuate the latent heat absorbed or released by the phase change.

Alboussière et al. (2010) have shown that the rate $V$ of phase change is proportional to the local topography $h$,

$V=\frac{h}{\tau_{\phi}}$,

with melting when the topography is positive, and crystallization when it is negative. The phase change timescale $\tau_{\phi}$ is

$\tau_{\phi}=\frac{L}{c_{p} \bar{u} \rho g^{\prime} r_{\mathrm{ic}} \frac{d\left(T_{\mathrm{s}}-T_{\mathrm{ad}}\right)}{d p}} \sim\left(\frac{1221 \mathrm{~km}}{r_{\mathrm{ic}}}\right) \times 10^{3}$ year,

where $\bar{u} \sim 10^{-4} \mathrm{~m} \mathrm{~s}^{-1}$ is the mean convective velocity in the outer core. The magnitude of the dynamic topography can be estimated by equating the topographic stress, which is of order $\Delta \rho g h$, with the viscous stress associated with convection, which is of order $\eta U / l$, where $U$ and $l$ are typical velocity and length scales of the convection. This gives

$h \sim \frac{\eta}{\Delta \rho g l} U=\frac{r_{\mathrm{ic}}}{l} \tau_{\eta} U$,

where $\tau_{\eta}=\eta /\left(\Delta \rho g r_{\text {ic }}\right)$ is the timescale of viscous relaxation. Combining eqs (33) and (35), the ratio of the phase change rate to the convective velocity is

$\frac{V}{U} \sim \frac{r_{\text {ic }}}{l} \frac{\tau_{\eta}}{\tau_{\phi}} \sim \frac{r_{\text {ic }}}{l}\left(\frac{\eta}{10^{18} \mathrm{~Pa} \mathrm{~s}}\right)\left(\frac{1221 \mathrm{~km}}{r_{\mathrm{ic}}}\right) \times 10^{-2}$.

This suggests that the phase-change at the ICB would have a small effect on the style and vigour of convection if the viscosity is small ( $\left.\lesssim 10^{18} \mathrm{~Pa} \mathrm{~s}\right)$, but would be important if the viscosity is larger. It has been recently proposed that convection in the inner core might take the form of a convective translation, with melting on one side of the inner core and crystallization on the other side (Monnereau et al. 2010; Alboussière et al. 2010). This is a 'high viscosity' regime which is expected to be dominant if the inner core viscosity is higher than about $10^{18} \mathrm{~Pa} \mathrm{~s}$ (Alboussière et al. 2010). In the limit of small viscosity however, convective stresses are too small to sustain a significant topography, and phase change is negligible. We focus here on this low viscosity limit, and, as a 
first step, ignore convectively induced phase change at the ICB. Simulations with topography-induced phase change at the ICB will be presented in a future paper.

\subsection{Taking into account the inner core growth}

Following Deguen \& Cardin (2009), we account for inner core growth by scaling lengths by $r_{\text {ic }}(t)$, thus transforming the problem from a moving boundary problem into a fixed boundary problem (Crank 1984) with $\tilde{r}=r / r_{\text {ic }}(t) \in[0,1]$. The time derivatives in the new coordinate system writes

$$
\left.\frac{\partial}{\partial t}\right|_{\tilde{r}}=\left.\frac{\partial}{\partial t}\right|_{r}-\left.\left.\frac{\partial \tilde{r}}{\partial t}\right|_{r} \frac{\partial}{\partial \tilde{r}}\right|_{t}=\left.\frac{\partial}{\partial t}\right|_{r}+\left.\tilde{r} \frac{u_{\text {ic }}}{r_{\text {ic }}} \frac{\partial}{\partial \tilde{r}}\right|_{t} .
$$

This introduces a radial advection term in the entropy and solute conservation equations, which accounts for the apparent inward transport of matter in the new reference frame; no similar term is introduced in the momentum conservation equation as long as inertia is negligible.

\subsection{Non-dimensionalization}

Time, lengths, fluid velocity and pressure are scaled by $\tau_{\text {ic }}, r_{i c}(t)$, $\kappa / r_{i c}(t)$ and $\eta \kappa / r_{i c}^{2}(t)$, respectively. The potential temperature is scaled by $S(t) r_{\mathrm{ic}}^{2} /(6 \kappa)$ and we introduce a scaled composition defined as $\chi=\left(c-D c_{0}^{l}\right) / \Delta c$, where $\Delta c=c\left[r_{\mathrm{ic}}(t)\right]-D c_{0}^{l} 111$.

The conservation of momentum and mass are expressed in nondimensional form as

$0=-\nabla p+\left(R a \Theta-R a_{\chi} \chi\right) \mathbf{r}+\nabla^{2} \mathbf{u}, \quad \nabla \cdot \mathbf{u}=0$,

where $\mathbf{r}=r \mathbf{e}_{\mathbf{r}}$ is the radius vector, and where the thermal Rayleigh number $R a$ and the chemical Rayleigh number $R a_{\chi}$ are defined as

$R a(t)=\frac{\rho g\left(r_{\mathrm{ic}}\right) \alpha S r_{\mathrm{ic}}^{5}}{6 \eta \kappa^{2}}$ and $R a_{\chi}(t)=\frac{\rho g\left(r_{\mathrm{ic}}\right) \beta \Delta c(t) r_{\mathrm{ic}}^{3}}{\eta \kappa}$

The equation of conservation of entropy [eq. (4)] is expressed in non-dimensional form, using eq. (37), as

$\xi \frac{\partial \Theta}{\partial t}=\nabla^{2} \Theta-(\mathbf{u}-P e \mathbf{r}) \cdot \nabla \Theta+6-\left(\xi \frac{\dot{S} \tau_{\text {ic }}}{S}+2 P e\right) \Theta$,

where the Peclet number $P e$ and the parameter $\xi$ are defined as

$P e(t)=\frac{r_{\mathrm{ic}}(t) u_{\mathrm{ic}}(t)}{\kappa} \quad$ and $\quad \xi(t)=\frac{r_{\mathrm{ic}}^{2}(t)}{\kappa \tau_{\mathrm{ic}}}$.

Similarly, the equation of conservation of solute is written in nondimensional form as

$\xi \frac{\partial \chi}{\partial t}=L e^{-1} \nabla^{2} \chi-(\mathbf{u}-P e \mathbf{r}) \cdot \nabla \chi-\xi \frac{\dot{\Delta} c \tau_{\text {ic }}}{\Delta c} \chi$,

where the Lewis number $L e$ is defined as

$L e=\frac{\kappa}{\kappa_{c}}$,

where $\kappa_{c}$ is the compositional diffusivity. The last terms on the right hand sides of eqs (40) and (41), proportional to $\Theta$ and $\chi$, respectively, appear because the temperature and compositional scales are time-dependent.

The ICB is treated as a shear stress free surface, and the boundary conditions at the ICB for the temperature and solute concentration are $\Theta\left(r_{\text {ic }}\right)=0$ and $\chi\left(r_{\text {ic }}\right)=1$.
The evolution of the time-dependent non-dimensional parameters is calculated at each time step. The core energy balance [eq. (15)] gives $r_{\text {ic }}$ and $u_{\text {ic }}$ as a function of time, eq. (18) gives the evolution of $S$ and eq. (27) gives $\Delta c$ as a function of time. The evolution of $R a, R a_{\chi}, P e$ and $\xi$ is then calculated from $r_{\text {ic }}, u_{\text {ic }}, S$ and $\Delta c$.

\subsection{Numerical method}

Eqs (38)-(41) are solved in 3-D with shear stress free conditions and uniform temperature and composition at the ICB. We use a spherical harmonic expansion for the horizontal dependence and a finite difference scheme in the radial direction. The non-linear part of the advection terms in eqs (40) and (41) is evaluated in the physical space at each time step (the linear radial advection term due to inner core growth is treated in the spectral domain). A semi-implicit Crank- Nickolson scheme is implemented for the time evolution of the linear terms and an Adams-Bashforth procedure is used for the non-linear terms. We typically use 128 radial points, with a finer grid below the ICB, and a spherical harmonic truncation at degree 64 .

\section{NUMERICAL RESULTS}

Given the number of free parameters involved and the large uncertainties associated with most of them, we will not attempt here a systematic parameter study. Rather, we will present a few representative examples chosen to highlight the different scenarios possible, and discuss qualitatively their implications in terms of seismological observations. Simulations without compositional stratification (purely thermal convection) are presented in Section 5.1. Simulations of thermochemical convection with a stabilizing chemical stratification are presented and discussed in Section 5.2.

In all the simulations discussed below, a thermal conductivity value of $k=36 \mathrm{~W} \mathrm{~m}^{-1} \mathrm{~K}^{-1}$ (Stacey \& Davis 2008) has been assumed, which, with $c_{p}=800 \mathrm{~J} \mathrm{~kg}^{-1} \mathrm{~K}^{-1}$ and $\rho=13000 \mathrm{~kg} \mathrm{~m}^{-3}$, gives $\kappa=3.5 \times 10^{-6} \mathrm{~m}^{2} \mathrm{~s}^{-1}$ and $\tau_{\kappa}=2.3 \mathrm{~Gy}$. Eq. (17) shows that the locus of the $S(t)=0$ curve is a function of $d T_{\mathrm{s}} / d T_{\mathrm{ad}}, r_{i c}$ and $\tau_{\text {ic }} / \tau_{\kappa}$ only, so that for larger thermal conductivities, similar behaviour would be found for smaller inner core age. The problem is not exactly self-similar however since the value of $S$, and hence the vigour of convection, depends additionally on $\kappa$. Numerical simulations with higher values of $\kappa$ show that the effect is small, and we thus restrict our discussion to the case $k=36 \mathrm{~W} \mathrm{~m}^{-1} \mathrm{~K}^{-1}$. For thermochemical simulation, the Lewis number is taken as $10^{3}$.

\subsection{Thermal convection}

Thermal convection in the inner core is transient. Both the radius of the inner core and the thermal forcing $S$ evolve continuously-and significantly - during the inner core history, which results in large variations in the Rayleigh number. The thermal forcing $S$ being a decreasing function of time, it is clear that, even if initially positive, it will become negative at some time during the inner core history, at which point convection would cease. Here, we will first present some numerical simulations in a situation were the inner core would still be in a convecting state today, and discuss the style of convection and give scaling laws for relevant variables. Simulations for which the inner core convects early in its history but is now quiescent are presented and discussed in a second step. 


\section{a. $\tau_{\text {ic }}=1.1 \mathrm{~Gy}, \eta=10^{18}$ Pa.s, $k=36 \mathrm{~W} \cdot \mathrm{m}^{-1} \cdot \mathrm{K}^{-1}$.}

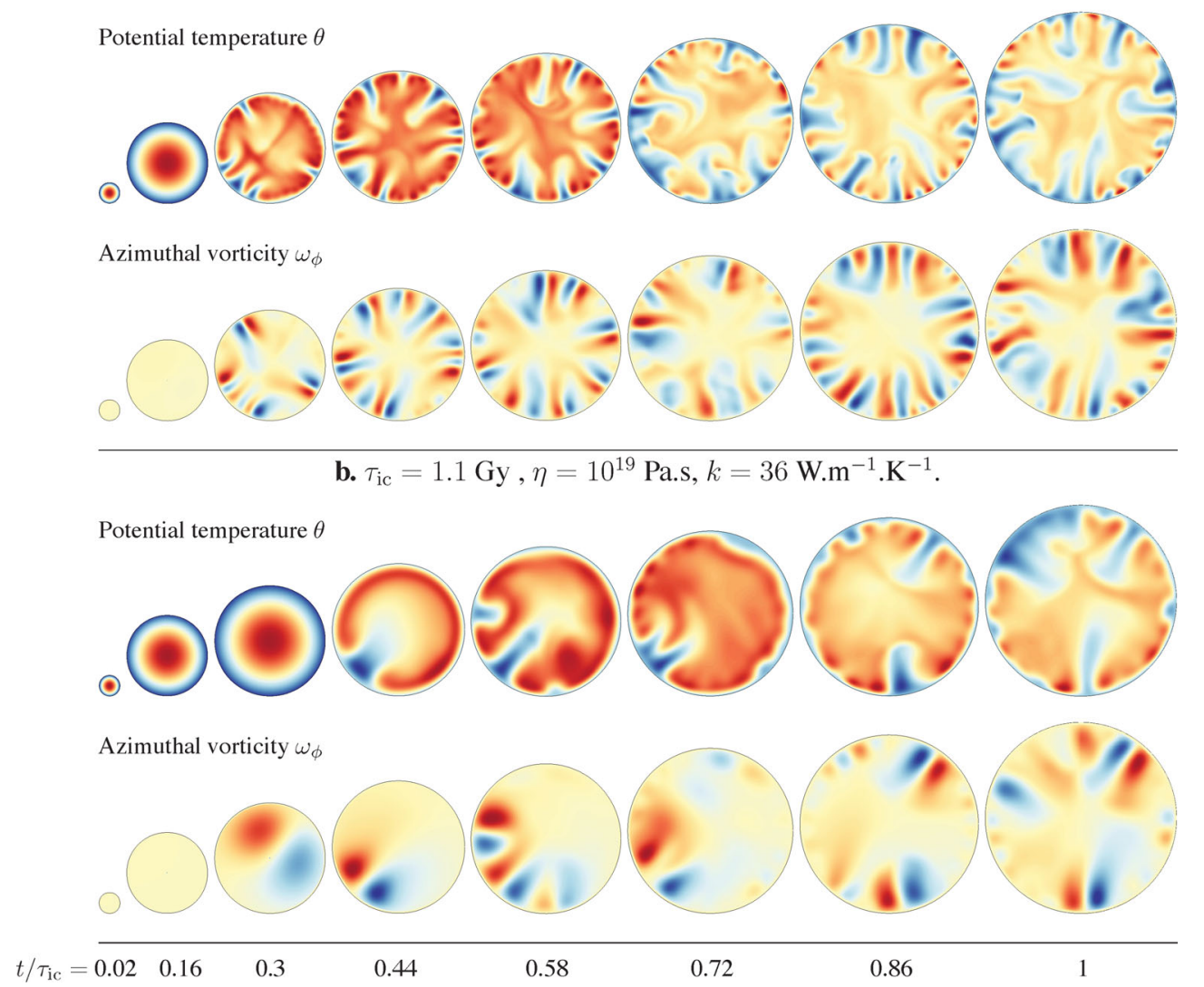

Figure 5. Time-series of snapshots of potential temperature and azimuthal vorticity in an arbitrary cross-section. Positive vorticity is red, negative vorticity blue. Each map has been drawn with its radius in proportion to the inner core radius at the time of the snapshot. Both simulations with $\tau_{\text {ic }}=1.1 \mathrm{~Gy}$ and $k=36$ $\mathrm{W} \mathrm{m}^{-1} \mathrm{~K}^{-1}$, and viscosities $\eta=10^{18} \mathrm{~Pa} \mathrm{~s}$ (a) and $\eta=10^{19} \mathrm{~Pa} \mathrm{~s}(\mathrm{~b})$. The non-dimensional time corresponding to each snapshot is shown in the bottom row.

\subsubsection{Ongoing thermal convection - phenomenology and scaling}

If $\mathcal{T}_{\text {ic }}$ is small enough (smaller than $\sim 0.8$ according to Fig. 3a), then $S$ remains positive during all the inner core history. With $k=$ $36 \mathrm{~W} \mathrm{~m}^{-1} \mathrm{~K}^{-1}$ and $d T_{\mathrm{s}} / d T_{\mathrm{ad}}=1.65$, this corresponds to an inner core younger than about $1.2 \mathrm{~Gy}$, or a CMB heat flux larger than $7.7 \pm$ 5.0 TW [using Labrosse (2003)'s estimate of $E_{\text {tot }}$ and associated uncertainties]. Thermal convection should then develop provided that the Rayleigh number is super-critical. The ratio of the Rayleigh number to the critical value for convection is approximately equal to

$\frac{R a}{R a_{c}} \simeq\left(\frac{10^{18} \mathrm{~Pa} \mathrm{~s}}{\eta}\right)\left(\frac{S}{10 \mathrm{~K} \mathrm{~Gy}^{-1}}\right)\left(\frac{r_{\mathrm{ic}}}{1221 \mathrm{~km}}\right)^{6} \times 250$,

so we can expect that the inner core would be likely to convect, provided that $S>0$, if its viscosity is smaller than $\sim 10^{20} \mathrm{~Pa}$ s.

Fig. 5 shows a suite of snapshots of the potential temperature and vorticity fields during the growth of the inner core, in the same arbitrary cross-section, for an inner core 1.1 Gy old, and two values of the viscosity, $\eta=10^{18} \mathrm{Pas}$ (Fig. 5a), and $\eta=10^{19} \mathrm{Pas}$ (Fig. 5b). After the Rayleigh number reaches the critical value for convection, the first event is always a degree 1 overturn which evacuates the superheat accumulated in the inner core during its early growth. The growth rate of the instability can be quite small, and the time at which convective motion becomes large (say, larger than the velocity of the ICB) can be significantly delayed after the Rayleigh number becomes super-critical. The pattern of flow then rapidly evolves toward smaller scales as the Rayleigh number increases. The convection regime is typical of high Rayleigh number internally heated convection, with narrow plumes sinking from a thermal boundary layer below the ICB and a passive rising return flow (McKenzie et al. 1974; Weinstein \& Olson 1990; Parmentier et al. 1994; Parmentier \& Sotin 2000).

The scaling theory of Parmentier \& Sotin (2000) for infinite Prandtl number, high Rayleigh number internally heated convection suggests that in steady state, the thickness of the boundary layer, $\delta$, and the potential temperature drop across the boundary layer, $\Delta \Theta$, should both scale as $R a^{-1 / 4}$, while the convective velocity scale as $R a^{1 / 2}$. As shown in Fig. 6, the dependence on $R a$ of the root mean square of the velocity, $u_{\text {rms }}$, the mean potential temperature in the inner core, $\Delta \Theta$, (and the root mean square of temperature fluctuations, $\delta \Theta_{\text {rms }}$ ) are relatively well described by Parmentier \& Sotin (2000)'s scaling when convection is well-developed. The agreement with the predicted scaling for the thermal boundary layer thickness $\delta$ is poor however. The best fits of our numerical results for $R a>$ $10^{7}$ give, with variables given in dimensional form,

$$
\begin{aligned}
& u_{\mathrm{rms}} \simeq 0.04 \frac{\kappa}{r_{\mathrm{ic}}} R a^{0.51}, \\
& \delta \Theta_{\mathrm{rms}} \simeq 1.5 \frac{S r_{\mathrm{ic}}^{2}}{6 \kappa} R a^{-0.27}, \\
& \Delta \Theta \simeq 3.8 \frac{S r_{\mathrm{ic}}^{2}}{6 \kappa} R a^{-0.27},
\end{aligned}
$$



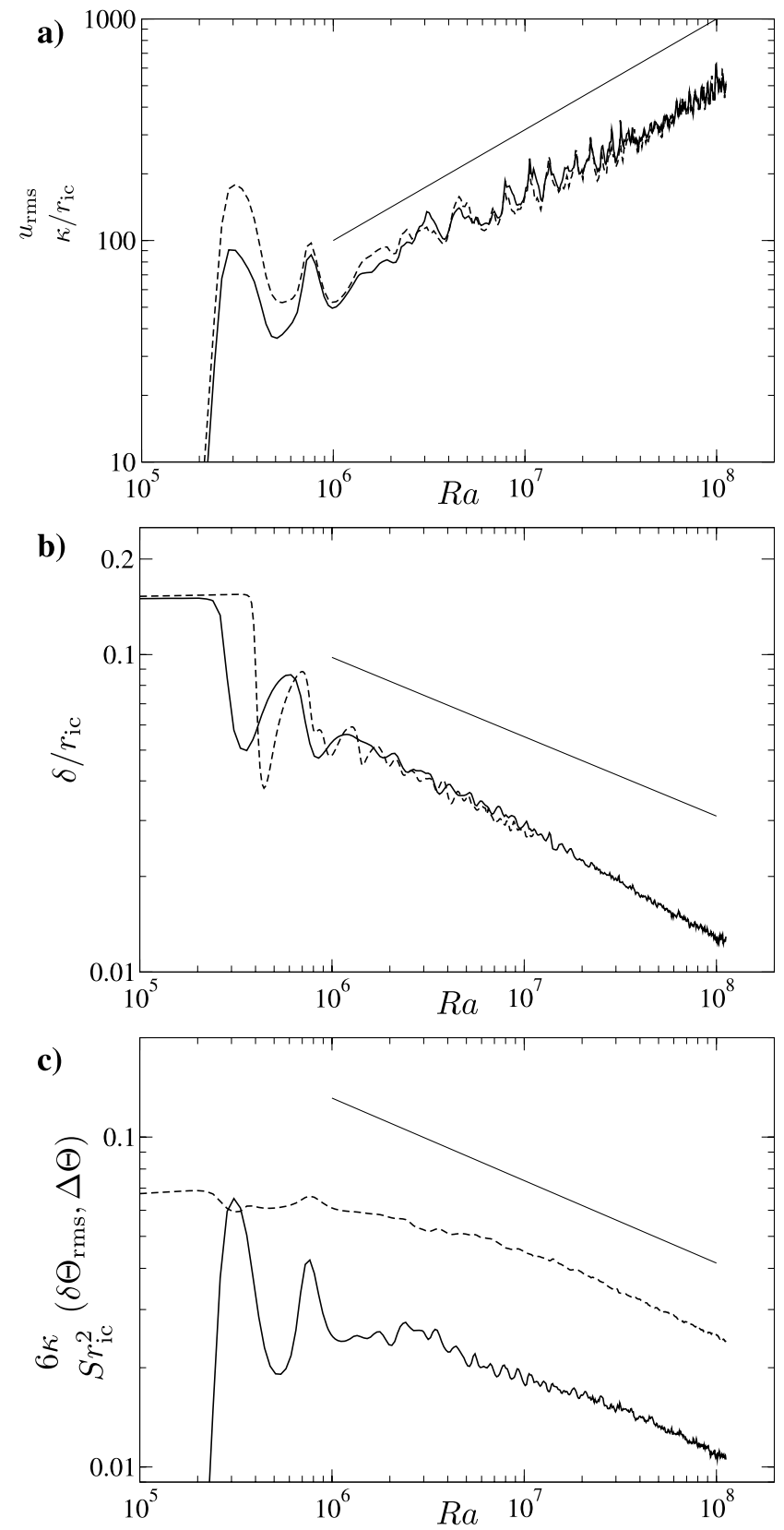

Figure 6. (a) Mean radial velocity (black solid line) and horizontal velocity (black dashed line) as a function of the Rayleigh number. The thin black line has a 1/2 slope. (b) Boundary layer thickness as a function of $R a$. The thin black line has a $-1 / 4$ slope. (c) RMS temperature fluctuations in the inner core (black solid line), and mean potential temperature (black dashed line). The thin black line has a $-1 / 4$ slope.

$\delta \simeq 6.4 r_{\text {ic }} R a^{-0.33}$

Departures from the scaling predicted by Parmentier \& Sotin (2000) are likely to come primarily from the fact that convection in the inner core is transient and may not be in a statistical quasisteady state. This can be seen as follows. In quasi-steady state, the heat flow at the ICB, $Q_{\text {icb }}=4 \pi r_{\text {ic }}^{2} \bar{q}$ should almost exactly balance the superadiabatic heat production within the inner core, which is $\frac{4}{3} \pi r_{\text {ic }}^{3} \rho c_{p} S$. Thus $\bar{q}$ should be independent of $R a$ and be

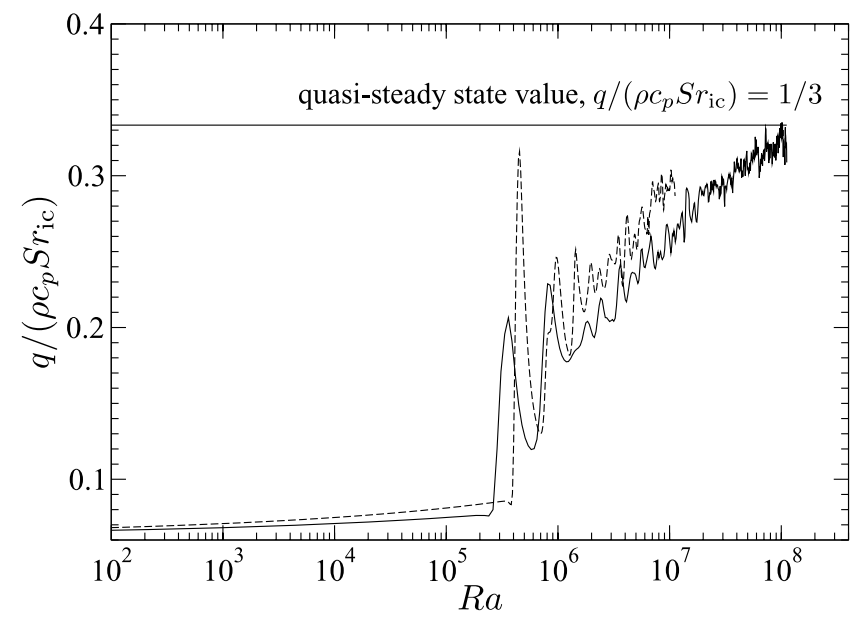

Figure 7. Normalized heat flux $\bar{q}$ as a function of $R a$ for several simulations with $\tau_{\text {ic }}=1.1$ Gy and $\eta=10^{17} \mathrm{~Pa} \mathrm{~s}$ (continuous black line), and $\eta=10^{18}$ Pa s (dashed black line).

equal to

$\bar{q}_{\text {steady state }}=\frac{1}{3} \rho c_{p} S r_{\text {ic }}$.

Fig. 7 shows $\bar{q}$ (normalized by $\rho c_{p} S r_{\text {ic }}$ ) as a function of $R a$ for several simulations. A thermal quasi-steady state is only reached at the highest Rayleigh number of our simulations, for $R a$ larger than $\sim 10^{8}$. A direct consequence can be seen from the fact that the ICB heat flux scales as

$\bar{q} \sim k \frac{\Delta \theta}{\delta}$.

Since in quasi-steady state, $\bar{q}$ is independent of $R a$, then $\Delta \theta$ and $\delta$ should have the same scaling in $R a$ (as is indeed the case in Parmentier \& Sotin (2000)'s scaling). This is not true if the convection is not in a quasi-steady state however, and this may partially explain the poor agreement of our measurements of $\delta$ and the theory.

An additional complexity comes from the fact that the ICB migrates as a result of solidification. In Howard's view of high Rayleigh number convection, the thermal boundary layer grows by conduction until it becomes unstable and is swept away by cold plumes that develop from the boundary layer instability. With a crystallizing boundary, however, the thermal boundary layer grows also by the addition of cold newly solidified material. An infinitely fine boundary layer would grow in a time $\delta t$ to a thickness $\sim \sqrt{\kappa \delta t}+u_{\text {ic }} \delta t$. The relative importance of the two effect depends on the magnitude of a local Peclet number defined as $P e_{\delta}=\delta u_{\text {ic }} / \kappa$. The ratio of $\sqrt{\kappa \delta t}$ to $u_{\text {ic }} \delta t$ for a boundary layer of thickness $\delta$ is equal to

$$
\frac{\sqrt{\kappa \delta t}}{u_{\mathrm{ic}} \delta t}=\frac{2}{\sqrt{1+4 P e_{\delta}}-1} .
$$

$P e_{\delta}$ is usually smaller than one in our simulations when convection is developed, but is not very small $(\sim 0.1-0.5)$, for which $(\sqrt{\kappa \delta t}) /\left(u_{\mathrm{ic}} \delta t\right)$ is between 2.7 and 10 , so it seems plausible that the dynamics of the boundary layer is slightly affected by the growth of the inner core.

\subsubsection{Cessation of convection}

If the inner core is slightly older $\left(1 \lesssim \mathcal{T}_{\text {ic }} \lesssim 2\right.$ according to Fig. 3a), then $S$ is initially positive but becomes negative at some point in the 
a. $\tau_{\text {ic }}=1.45 \mathrm{~Gy}, \eta=10^{17}$ Pa.s, $k=36 \mathrm{~W} \cdot \mathrm{m}^{-1} \cdot \mathrm{K}^{-1}$.

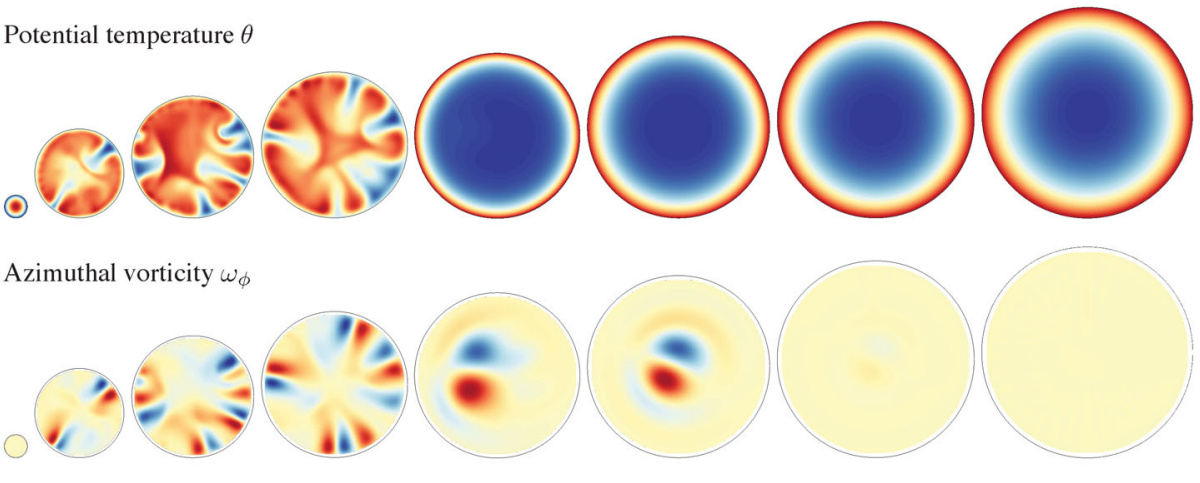

b. $\tau_{\text {ic }}=1.45 \mathrm{~Gy}, \eta=10^{18}$ Pa.s, $k=36 \mathrm{~W} \cdot \mathrm{m}^{-1} \cdot \mathrm{K}^{-1}$.

Potential temperature $\theta$

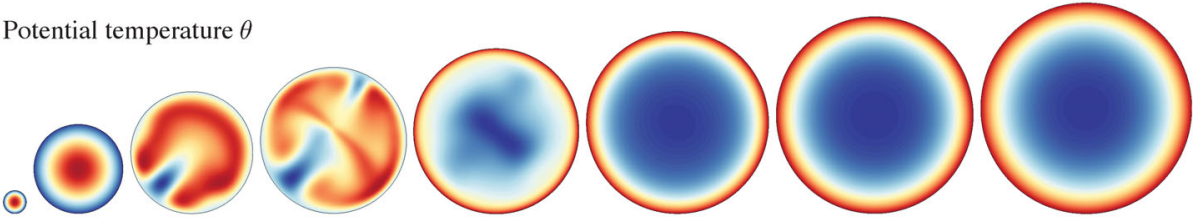

Azimuthal vorticity $\omega_{\phi}$
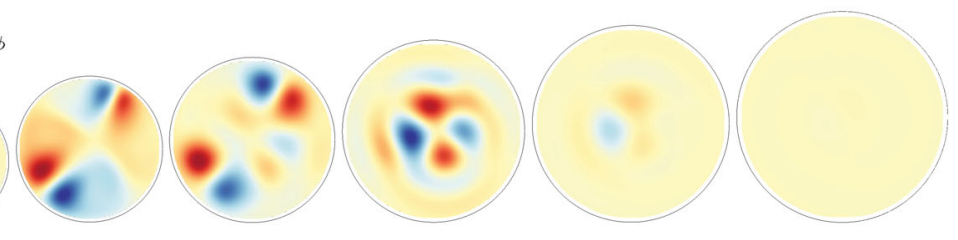

c. $\tau_{\text {ic }}=1.45 \mathrm{~Gy}, \eta=10^{19}$ Pa.s, $k=36 \mathrm{~W} \cdot \mathrm{m}^{-1} \cdot \mathrm{K}^{-1}$.

Potential temperature $\theta$
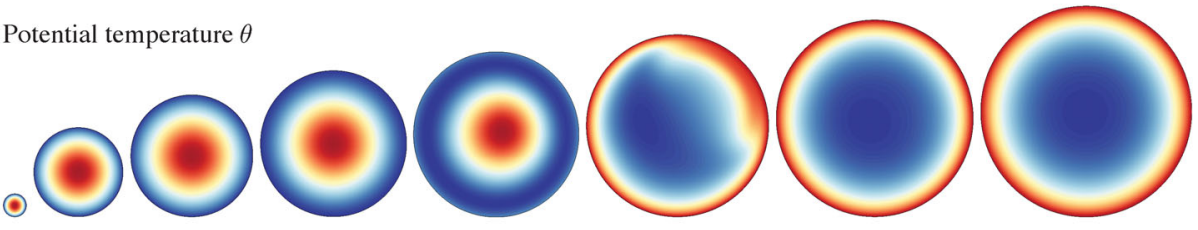

Azimuthal vorticity $\omega_{\phi}$
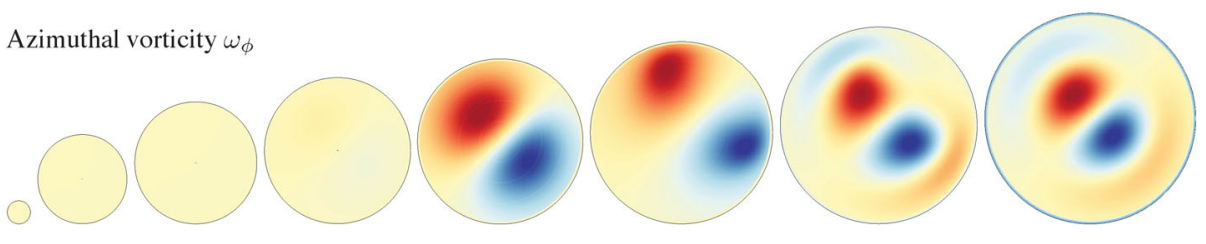

$t / \tau_{\text {ic }}=0.02 \quad 0.16 \quad 0.3$

0.44

0.58

0.72

0.86

1

Figure 8. Time-series of snapshots of potential temperature and azimuthal vorticity in an arbitrary cross-section. Positive vorticity is red, negative vorticity blue. Each map has been drawn with its radius in proportion to the inner core radius at the time of the snapshot. The non-dimensional time corresponding to each snapshot is shown in the bottom row.

inner core history. Fig. 8 shows a suite of snapshots of the potential temperature and vorticity fields during the growth of the inner core, in the same arbitrary cross-section, for an inner core 1.45 Gy old, and $\eta=10^{17} \mathrm{~Pa} \mathrm{~s}$ (Fig. 8a), $\eta=10^{18} \mathrm{~Pa} \mathrm{~s}$ (Fig. 8b), and $\eta=10^{19} \mathrm{~Pa}$ $\mathrm{s}$ (Fig. 8c). The exact times of convection initiation and cessation are only approximately predicted by the criterion on $S$ because: (i) the growth rate of the initial instability is a function of viscosity and (ii), the rate of heat extraction from the inner core is a function of the Rayleigh number when the convection is not in thermal quasisteady state (Fig. 7). As a result, convection starts and stops earlier for smaller viscosity, as is apparent in Fig. 8. If convection is in a quasi-steady state however, the time at which convection stops should be close to the time at which $S$ becomes negative.
A difficulty of thermal convection for explaining the inner core structure is that it is not clear why a convective pattern aligned with the rotation axis should be preferred. A possibility recently advocated by Buffett (2009) is that the centrifugal force associated with the Earth rotation may align the lowest order convective mode with the rotation axis. The centrifuge acceleration introduces a small anisotropic forcing (gravity, and ellipticity of the inner core) and promotes a $\mathrm{N}-\mathrm{S}$ symmetry of the convection pattern when the degree $\ell=1$ is dominant. Buffett (2009) hypothesized that when convection decreases in vigour, the last active mode would be a degree one convective pattern aligned with the rotation axis. Our model does not include the effect of rotation on the gravity potential and therefore cannot be used to test whether the convection 
would indeed align with the rotation axis. However, we can investigate in what conditions a low order texturation pattern might result from inner core convection. Specifically, a potential difficulty of this mechanism is that the amount of strain due to this late stage low order convective motion might be small, because (i) convection would be only slightly supercritical and would therefore be very sluggish, and (ii) low order modes might be active and dominant during only a small amount of time.

To investigate this, we define a velocity scale $u_{\ell}$ associated with each degree $\ell$ by calculating the root mean square of the components of degree $\ell$, order $m$,

$u_{\ell}=\sqrt{\frac{1}{\ell+1} \sum_{0 \leq m \leq \ell}\left(\bar{u}_{\ell}^{m}\right)^{2}}$,

where $\bar{u}_{\ell}^{m}$ is the mean value of the degree $\ell$ order $m$ component of the flow in the inner core. From this, a rough estimate of the strain rate associated with each degree $\ell$ is given by

$\dot{\epsilon}_{\ell} \sim \frac{u_{\ell}}{r_{\text {ic }} / \ell}$,

where $r_{\mathrm{ic}} / \ell$ is the typical lengthscale of degree $\ell$ flow components. The cumulated strain over a given period of time can then be estimated by integrating in time the expression of $\dot{\epsilon}_{\ell}$ given above. This is a quite simplistic view of the question of texturation, which is in general a non-linear mechanism - the final texture is not the sum of the texture which would have developed if each component of the flow was taken separately. However, this should give an idea of the likelihood of the development of a large scale texture, and of the typical length scale of texture variations.

The evolution of $u_{\ell}$ for $\ell \in[1,5]$ for the simulations presented in Fig. 8 is plotted in Fig. 9. Also shown in Fig. 9(d) are the values of the Rayleigh number based on the value of $S(t)$ given in eq. (39) (black lines), and another Rayleigh number based on the actual potential temperature difference between the centre of the core and the ICB (grey lines). Fig. 9(d) shows that the decrease in Rayleigh number when $S$ becomes negative is very abrupt, $R a$ decreasing from its quasi-steady value to zero in only a few tens of million years. The time during which the degree one mode is the only unstable mode is very short, less than $\sim 10 \mathrm{My}$. This is due to the fact that $R a \propto$ $S r_{\text {ic }}^{6}$ and that $S$ is a relatively weak function of $r_{\text {ic }}$ compared to $r_{\text {ic }}^{6}$. Furthermore, alignment of the degree one convective mode with the axis of rotation requires that this cessation timescale is long enough compared to the convective overturn timescale, which, using eq. (43), is about

$$
\begin{aligned}
\tau_{c} & =\frac{r_{\mathrm{ic}}}{u_{\mathrm{rms}}}=\frac{r_{\mathrm{ic}}^{2}}{\kappa 0.04 R a^{1 / 2}} \\
& =\left(\frac{R a}{10^{6}}\right)^{-1 / 2} \times 180 \mathrm{My} .
\end{aligned}
$$

It therefore does not seem likely that the low order convective modes would have enough time to align with the rotation axis if having an initially different orientation.

In our simulations, the cessation timescale of convection is small compared to a typical convective overturn timescale. The consequence is that the perturbations of the thermal field are basically frozen in when the Rayleigh number becomes subcritical. The resulting density distribution is out of equilibrium, and the inner core then relaxes toward a state of hydrostatic equilibrium. The total strain associated with each flow component therefore depends on the amplitude of the density field heterogeneities just before the Rayleigh number becomes subcritical.
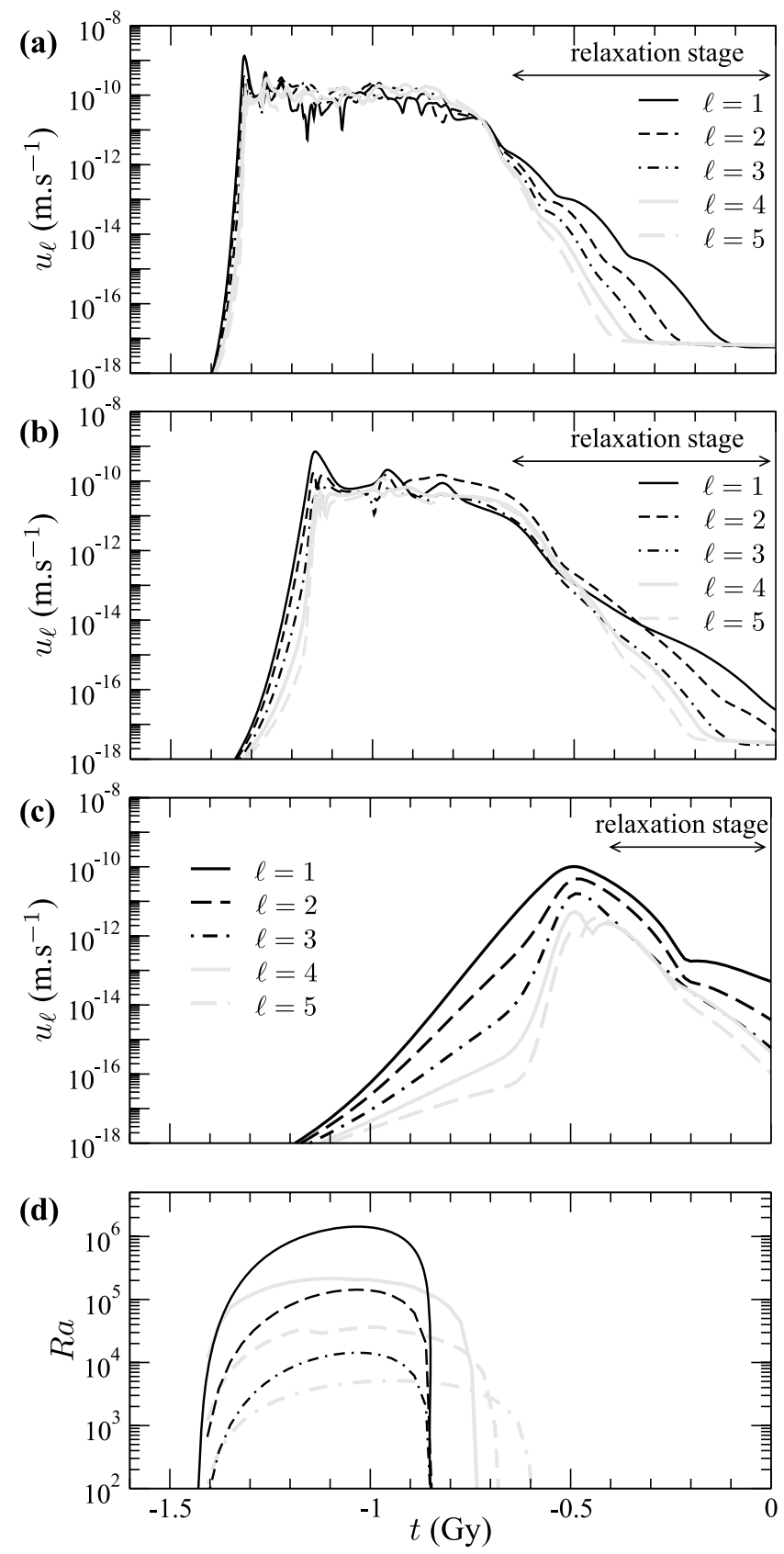

Figure 9. (a), (b) and (c): Velocity scale $u_{\ell}$, as defined in eq. (50), as a function of time for three simulations with $\tau_{\text {ic }}=1.45$ Gy and $\eta=10^{17} \mathrm{~Pa}$ s (a), $\eta=10^{18} \mathrm{~Pa} \mathrm{~s}$ (b) and $\eta=10^{19} \mathrm{~Pa} \mathrm{~s}$ (c). (d): Rayleigh number, as defined by eq. (39) (black lines), and calculated from the mean inner core potential temperature, $R a_{\theta}=\left(\alpha \rho g^{\prime} \Delta \Theta r_{\mathrm{ic}}^{4}\right) /(\eta \kappa)$ (grey lines), as a function of time, for $\eta=10^{17} \mathrm{~Pa} \mathrm{~s}$ (continuous lines), $10^{18} \mathrm{~Pa} \mathrm{~s}$ (dashed lines) and $10^{19} \mathrm{~Pa} \mathrm{~s}$ (dash-dotted lines).

As shown in Fig. 9, the degree one always becomes dominant at some point (thermal diffusion tends to weaken small scale thermal heterogeneities), but the energy associated is small. A rough estimate of the cumulated strain associated with an order $\ell$ component of the flow over a given period of time can be calculated by integrating eq. (51) in time. This should give a meaningful result when calculated over the relaxation period, because the pattern of the flow does not change over this period of time (although, again, caution should be used when interpreting the results because deformation texturing is a non-linear process). When this procedure is applied 


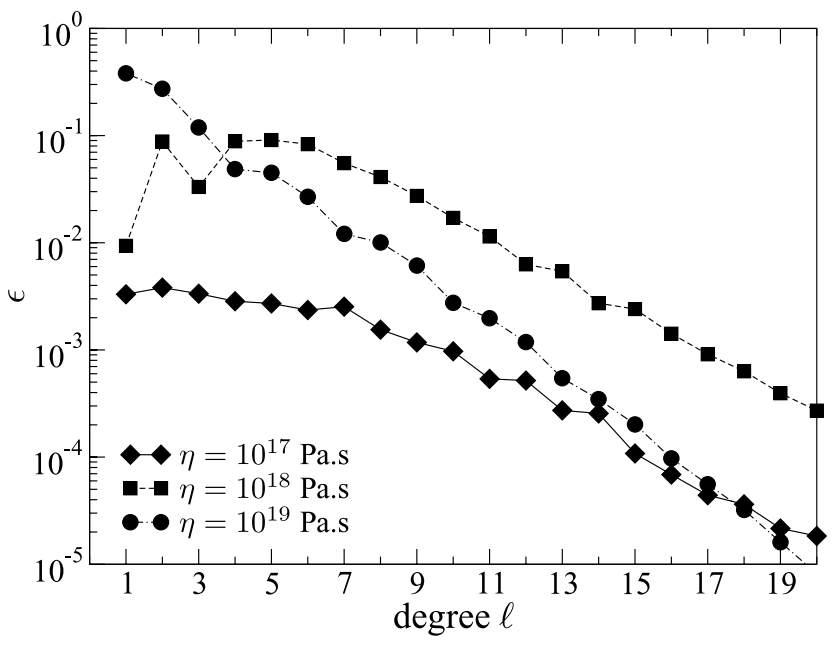

Figure 10. An estimate of the cumulated strain $\epsilon$ associated with each degree $\ell$ component of the flow during the relaxation phase. In the $\eta=$ $10^{19} \mathrm{~Pa}$ s case, the cumulated strain is calculated over the whole inner core history; for $\eta=10^{18} \mathrm{~Pa} \mathrm{~s}$ and $\eta=10^{17}$, the cumulated strain has been calculated over the last $700 \mathrm{My}$.

for each component of the flow, a spectrum of cumulated strain can be constructed, as shown in Fig. 10. For the case with the larger viscosity, $\eta=10^{19} \mathrm{~Pa} \mathrm{~s}$, the cumulated strain has been calculated over the whole inner core history, since there is only one convective overturn in this simulation, and the pattern of convection does not change. For each case, the strain associated with the relaxation phase is quite small. With $\eta=10^{19} \mathrm{~Pa} \mathrm{~s}$, the strain associated with the convective overturn is dominated by degree 1 components, and is of order 0.4 . This might be enough to induce a weak texture. In this case, since the degree 1 component of the flow is always dominant, it is plausible that the effect of the centrifugal acceleration is large enough to align the convection with the Earth's rotation axis. With a smaller viscosity, the strain associated with the relaxation phase is small, probably too small to result in a significant texture. The relaxation phase seems to be unlikely to significantly affect the texture developed previously. This suggests that a low order texturation pattern would be dominant only if the convection is dominated by low order components during most of the convection episode. This would be the case if the viscosity is $\gtrsim 10^{19} \mathrm{~Pa}$ s (Fig. 8c).

\subsection{Thermochemical convection}

We now turn to the question of the effect of a possible stabilization of convection by an adverse compositional gradient (Section 3). It has been proposed that the development of a stable compositional stratification can suppress convection even if the inner core is thermally unstable (Buffett 2000, 2009; Deguen \& Cardin 2009). Because the magnitude of the compositional stratification increases concomitantly to the inner core growth, it is possible that thermal convection starts early in the inner core history before being stabilized by the compositional stratification, which is a possible explanation for the origin of the innermost inner core (Buffett 2000, 2009; Deguen \& Cardin 2009). The argument is somewhat weakened by the realization that a decrease of the effective distribution coefficient during inner core growth may significantly affect the magnitude of the stratification in light elements in the inner core [Alboussière et al. (2010), and see Section 3], but the problem is still worth investigating. We discuss here simulations of thermochemi- cal convection in the case where the inner core has a superadiabatic temperature profile and a stabilizing compositional stratification.

It is well known that in classical double-diffusive convection (with aqueous solutions for example), convection can occur even if the net density gradient is stable, a consequence of the difference of diffusivity (e.g. Turner 1980). Linear stability analysis (Veronis 1965; Baines \& Gill 1969) shows that in the case of unstable thermal field and stable compositional field the critical Rayleigh number $R a_{c}$ for convective instability is given by

$R a_{c}=\frac{P r+L e^{-1}}{P r+1} R a_{\chi}+\left(1+\frac{1}{L e}\right)\left(1+\frac{1}{\operatorname{Pr} L e}\right) \frac{27 \pi^{4}}{4}$.

This expression for $R a_{c}$ is only valid for semi-infinite horizontal layers, and is not quantitatively exact for convection in a sphere, but should still give some useful guidance. It can be seen that the curve of marginal stability $R a_{c}=f\left(R a_{\chi}\right)$ has a slope smaller than 1 when $L e>1$. This implies that if $R a_{\chi}$ is large enough, the system can be unstable even if $R a / R a_{\chi}=\alpha \Delta T /|\beta \Delta c|<1$ [i.e. with a net density profile $\rho(\alpha \Theta+\beta c)$ which is stable]. However, the slope of the marginal stability curve tends toward 1 for $\operatorname{Pr} \gg 1$, which means that in the inner core, where $P r$ is likely to be larger than $10^{15}$, convection actually requires that the net density profile is unstable. Our simulations are in agreement with this prediction.

Fig. 11 shows snapshots (potential temperature, composition and azimuthal vorticity) from a simulation with $\tau_{\text {ic }}=1.2 \mathrm{~Gy}, \eta=10^{18}$ Pa s, a thermal conductivity $k=36 \mathrm{~W} \mathrm{~m}^{-1} \mathrm{~K}^{-1}$ and initial concentration of incompatible light element $(\mathrm{O})$ of $5.6 \mathrm{wt} . \%$ in the core. In this simulation, the age of the inner core and its thermal conductivity are such that the inner core is expected to be thermally unstable during the whole simulation. Interestingly, convection becomes progressively confined in the deepest part of the inner core, as can be seen in Figs 11 and 12(a). This results from the fact that the temperature and compositional profiles have different radial dependences, which implies that the relative contributions of temperature and composition to the density gradient is a function of radius. Since the chemical stratification is $\alpha r^{3}$ while the potential temperature is expected to be $\propto 1-r^{2}$ at first order, chemical stratification can stabilize the uppermost inner core while still allowing thermally driven convection in the deepest inner core. In the case shown here, large radial velocities are confined in an 'innermost inner core' of radius $\sim 800 \mathrm{~km}$. A stronger chemical stratification or a smaller value of the parameter $S$ (older inner core) results in a smaller convecting region.

A particularity of double-diffusive convection can be seen in the behaviour of a global Nusselt number defined as

$N u=1+\frac{\overline{q_{\text {adv }}}}{\overline{q_{\text {diff }}}}=1-\frac{\overline{u_{r} \Theta}}{\frac{\partial \Theta}{\partial r}}$,

where the overbar denotes the average over the inner core volume, $\overline{q_{\text {adv }}}$ is the average of the local advective heat flux, and $\overline{q_{\text {diff }}}$ is the average of the diffusive superadiabatic flux. As defined here, $\mathrm{Nu}$ compares the total superadiabatic heat flux to the diffusive superadiabatic heat flux; the contribution of diffusion along the adiabat is not taken into account with this definition. Fig. 12(b) shows the evolution with time of the global Nusselt number for the simulation shown in Fig. 11. It is interesting to note that the Nusselt number can be smaller than one early in the simulation, which means that there is at some time a net inward advection of heat. This is an expression of the double-diffusive nature of convection: Isocompositional surfaces are deformed by thermally driven convection, which gives rise to restoring compositional buoyancy forces. Since heat diffusion is fast compared to chemical diffusion, the magnitude 
$\tau_{\text {ic }}=1.2 \mathrm{~Gy}, \eta=10^{18} \mathrm{~Pa} . \mathrm{s}, k=36 \mathrm{~W} . \mathrm{m}^{-1} . \mathrm{K}^{-1}, c_{0}=5.6 \mathrm{wt} . \%$.
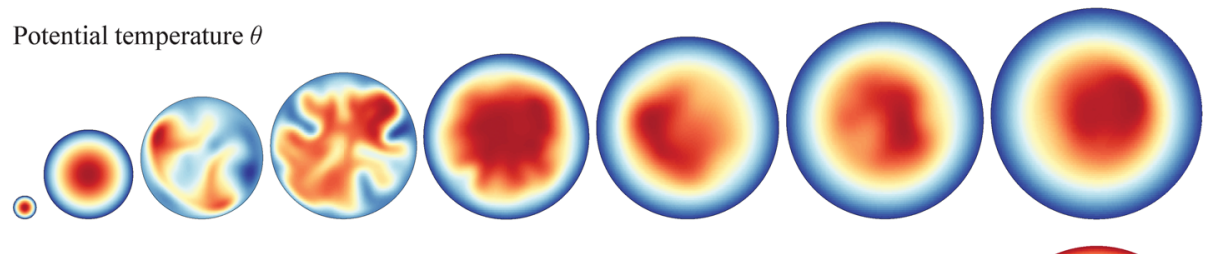

Composition $\chi$
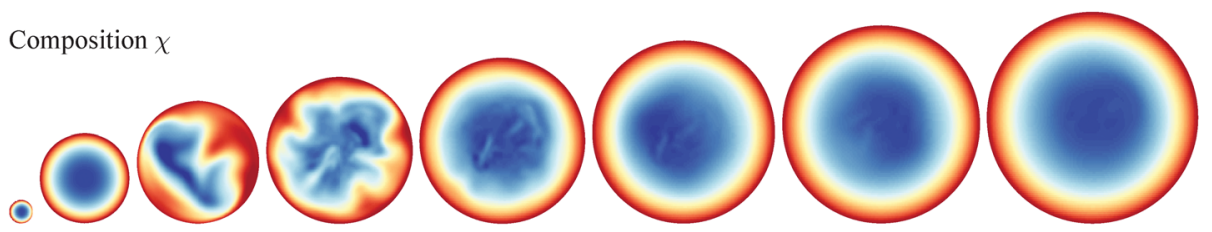

Azimuthal vorticity $\omega_{\phi}$
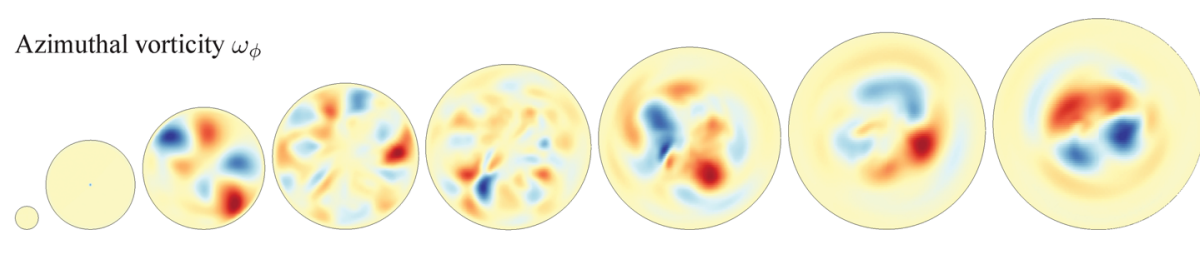

$t / \tau_{\text {ic }}=0.02 \quad 0.16$

0.3

0.44

0.58

0.72

0.86

1

Figure 11. Time-series of snapshots of potential temperature, composition and azimuthal vorticity in an arbitrary cross-section. Positive vorticity is red, negative vorticity blue. Each map has been drawn with its radius in proportion to the inner core radius at the time of the snapshot. Simulation with $\tau_{\text {ic }}=$ $1.2 \mathrm{~Gy}, k=36 \mathrm{~W} \mathrm{~m}^{-1} \mathrm{~K}^{-1}$, light element concentration in the outer core $c_{0}=5.6 \mathrm{wt} . \%$, and dynamic viscosity $\eta=10^{18} \mathrm{~Pa}$ s. The non-dimensional time corresponding to each snapshot is shown in the bottom row.

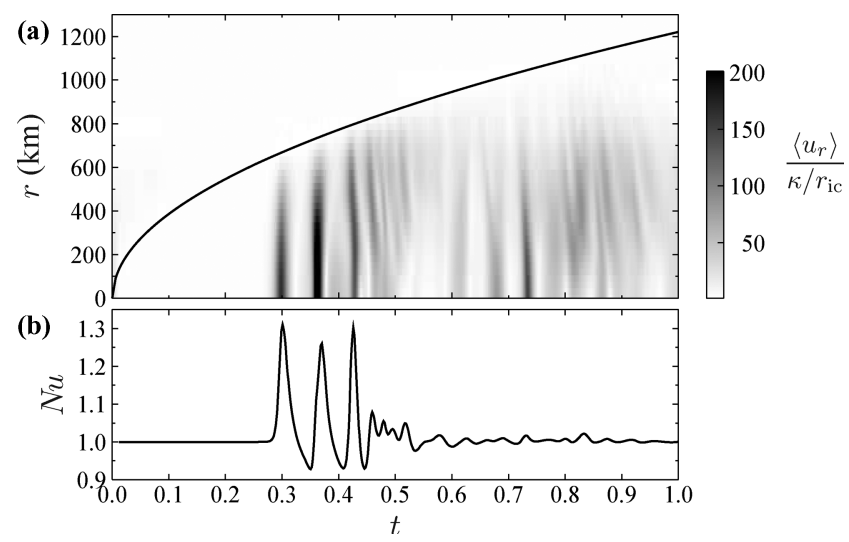

Figure 12. (a) Radial velocity averaged over spherical shells of radius $r$, $\left\langle u_{r}\right\rangle$, as a function of time and radius in the inner core. The black line represents the radius of the inner core as a function of time, for the simulation described in Fig. 11. In this simulation, large velocities are confined in an 'innermost inner core' of radius $\sim 800 \mathrm{~km}$. (b) Global Nusselt number as a function of non-dimensional time.

of temperature heterogeneities decreases much faster than compositional heterogeneities, and eventually becomes smaller, at which point the motion is reversed and chemical buoyancy transports heat back inward.

\section{DISCUSSION}

\subsection{Orientation of the convection}

A classically cited difficulty of thermal convection as a mechanism for producing the inner core anisotropy is the need of an additional mechanism to align convective patterns with the rotation axis (Sumita \& Bergman 2007; Buffett 2009). A second difficulty sug- gested by our simulations is that the flow is expected to be dominated by small scale motion and shows considerable time variability if the viscosity is smaller than $\sim 10^{19} \mathrm{~Pa}$ s. It seems rather unlikely that such convection can produce a large scale cylindrical anisotropy.

Aligning the convective patterns with the rotation axis requires the presence of some NS-aligned aspherical forcing in either body forces or boundary conditions. Buffett (2009) proposed that the centrifugal acceleration associated with the Earth's rotation can provide such a forcing and align the degree one flow component with the rotation axis, but it is not clear from our simulations that the last slightly super-critical stages of convection can produce cumulated stress large enough to produce an observable texture.

Preferential growth of the inner core in the equatorial belt (Yoshida et al. 1996) would produce boundary conditions with a NS axis of symmetry which may force, or at least favour, flow patterns with a NS axis of symmetry. The flow associated with the relaxation of a sustained ICB topography is weak however, with typical velocity of the order of or smaller than the inner core crystallization rate (Yoshida et al. 1996). It may therefore indeed help to align low order components of the convection with the rotation axis, but the degree one components would still be relatively weak compared to smaller scale flow components.

Another possibility is to envisage a coupling between convection and the magnetic field (Karato 1999; Buffett \& Wenk 2001). The magnetic field within the inner core results from the diffusion of the outer core magnetic field. High frequency temporal variations are filtered out by the skin effect, and diffusion ensures that the large scale features of the magnetic field dominate in the deep inner core. The magnetic field in the inner core is therefore expected to be a time averaged, lowpass filtered version of the magnetic field in the outer core, and is likely to display a North-South cylindrical symmetry and predominantly large scale features. One interesting aspect of the magnetic field is therefore that the Lorentz force would inject energy in the low order components of the convective flow, 
and might be able to sustain vigorous large scale motions which otherwise would not be favoured by natural convection.

The magnetic field is expected to alter significantly the pattern of motion if magnetic drag is a significant fraction of viscous drag. The largest contribution to the Lorentz force is expected to be that associated with the toroidal field $B_{\phi}$ (Karato 1999), which gives rise in the inner core to a poloidal Lorentz force field with cylindrical symmetry. The ratio of the Lorentz force $\mathbf{f}_{L}=\mu_{0}(\nabla \times \mathbf{B}) \times \mathbf{B}$ to the viscous force associated with the degree one component of the flow is of the order of

$$
\begin{aligned}
\frac{\left\|\mathbf{f}_{L}\right\|}{\left\|\eta \nabla^{2} \mathbf{u}\right\|_{l}} & \sim \frac{B_{\phi}^{2} r_{\mathrm{ic}}}{\mu_{0} \eta U}=25 \frac{B_{\phi}^{2} r_{\mathrm{ic}}^{2}}{\mu_{0} \eta \kappa} R a^{-1 / 2} \\
& \sim\left(\frac{B_{\phi}}{3 \mathrm{mT}}\right)^{2}\left(\frac{10^{16} \mathrm{~Pa} \mathrm{~s}}{\eta}\right)^{1 / 2}\left(\frac{10 \mathrm{KGy}^{-1}}{S}\right)^{1 / 2} \times 0.3,
\end{aligned}
$$

where $U \simeq 0.04 R a^{-1 / 2}$ [eq. (43)] is the velocity scale, and $\mu_{0}$ the magnetic permeability of vacuum. There is a range of geophysically plausible values of $B_{\phi}, S$ and $\eta$ for which the Lorentz force is of a magnitude comparable to that of the viscous force associated with the large scale flow. With a magnetic field intensity of a few $\mathrm{mT}$ (Christensen \& Aubert 2006; Gillet et al. 2010), the Lorentz force could be comparable in magnitude to viscous forces if $\eta \lesssim 10^{16}$ $\mathrm{Pa}$ s. If the toroidal field is as strong as $10 \mathrm{mT}$ at the ICB, Lorentz force could be of importance if $\eta \lesssim 10^{18} \mathrm{~Pa}$ s. It seems therefore plausible that the low order component of the convective flow can be aligned with the mean orientation of the magnetic field. This will be investigated in a future work.

\subsection{Thermally induced $V_{P}$ heterogeneities}

Convection induces lateral temperature heterogeneities which may results in observable $P$-wave velocity heterogeneities (Weber \& Machetel 1992). From the ab initio results of Vočadlo (2007), the relative variation of $V_{P}$ with temperature, $\left(\partial \ln V_{P} / \partial T\right)$, is of order $8 \times 10^{-5} \mathrm{~K}^{-1}$. Using the scaling from eq. (44) for the temperature perturbations (with the predicted exponent $-1 / 4$ substituted to -0.27 for simplicity), $P$-wave velocity variation associated with this thermal heterogeneities would be of order

$$
\begin{aligned}
\frac{\delta V_{P}}{V_{P}} & =\left(\frac{\partial \ln V_{P}}{\partial T}\right)_{P} \delta T \\
& \simeq 1.5\left(\frac{\partial \ln V_{P}}{\partial T}\right)_{P} \frac{S r_{\mathrm{ic}}^{2}}{6 \kappa} R a^{-1 / 4} .
\end{aligned}
$$

With $S=200 \mathrm{~K} \mathrm{~Gy}^{-1}$ and $\eta=10^{18} \mathrm{~Pa} \mathrm{~s}, \delta V_{P} / V_{P} \simeq 0.05$ per cent, which is probably close to an upper bound. A value two order of magnitude smaller is predicted if $S \simeq 10 \mathrm{~K} \mathrm{~Gy}^{-1}$ and $\eta=$ $10^{14} \mathrm{~Pa}$ s. For comparison, Garcia \& Souriau (2000) give an upper bound on lateral heterogeneity of $\sim 0.3$ per cent at length scales larger than $200 \mathrm{~km}$. Vidale \& Earle (2000) estimated that variations of $\sim 1$ per cent with a lengthscale $\sim 2 \mathrm{~km}$ are required to explain the $P K i K P$ codas they observed. Given the small effect of temperature anomalies we predict, it seems unlikely that temperature variations induced by thermal convection can be at the origin of $P$-wave heterogeneities of this magnitude.

\subsection{Partial melting below the ICB}

Decompression melting may occur below the ICB, in a way much similar as partial melting below oceanic ridges. As noted by Weber
\& Machetel (1992), the thermal gradient just below the ICB in a convective inner core may exceed the melting temperature gradient, in which case partial melting would occur in the boundary layer. This requires that

$-\left.\frac{\partial T}{\partial r}\right|_{\text {icb }} \geq-\left.\frac{\partial T_{\mathrm{s}}}{\partial r}\right|_{\text {icb }}$,

which can be rewritten as a function of the potential temperature $\Theta$ as

$-\left.\frac{\partial \Theta}{\partial r}\right|_{\mathrm{icb}} \geq\left.\frac{\partial T_{\mathrm{ad}}}{\partial r}\right|_{\mathrm{icb}}-\left.\frac{\partial T_{\mathrm{s}}}{\partial r}\right|_{\mathrm{icb}}$

Fig. 7 shows that the superadiabatic heat flux is bounded from above by $\frac{1}{3} \rho c_{p} S r_{\text {ic }}$, which implies that

$-\left.\frac{\partial \Theta}{\partial r}\right|_{\text {icb }} \leq \frac{1}{3} \frac{S r_{\text {ic }}}{\kappa}$

From eqs (60) and (61), we find that a necessary condition for partial melting is that

$S \geq 3 \kappa \frac{\rho g^{\prime} \gamma T}{K_{S}}\left(\frac{d T_{\mathrm{s}}}{d T_{\mathrm{ad}}}-1\right) \simeq\left(\frac{k}{36 \mathrm{~W} \mathrm{~m}^{-1} \mathrm{~K}^{-1}}\right) 40 \mathrm{~K} \mathrm{~Gy}^{-1}$.

Using eq. (8), this can be rewritten as a criterion for the present day inner core mean solidification rate,

$u_{\mathrm{ic}} \geq 3 \frac{\frac{d T_{\mathrm{s}}}{d T_{\mathrm{ad}}}}{\frac{d T_{\mathrm{s}}}{d T_{\mathrm{ad}}}-1} \frac{\kappa}{r_{\mathrm{ic}}} \simeq\left(\frac{k}{36 \mathrm{~W} \mathrm{~m}^{-1} \mathrm{~K}^{-1}}\right) 2.4 \times 10^{-11} \mathrm{~m} \mathrm{~s}^{-1}$.

With the core thermal history model and parameters of Labrosse (2003) and $k=36 \mathrm{~W} \mathrm{~m}^{-1} \mathrm{~K}^{-1}$, this requires that the inner core is younger than $\sim 0.65 \pm 0.08 \mathrm{~Gy}$, which requires a CMB heat flux larger than $14 \pm 9$ TW. Partial melting is possible, but requires a young inner core, and is not a necessary consequence of thermal convection. If the conditions for partial melting are met, partial melting will be localized in the thermal boundary layer which, according to our simulations, will have a thickness which may be a few tens of kilometres or smaller depending on the inner core Rayleigh number.

\section{CONCLUSION}

It is currently difficult to reach firm conclusions on the possibility of convection in the inner core. Our analysis shows that the range of estimates of the inner core age and on the critical age for purely thermal convection fully overlap, and thermal convection appears to be as likely as not. Radiogenic heating is probably a secondary issue for the thermal state of the inner core if radioactive elements partition weakly upon solidification, but may be of importance if they partition strongly. As shown in Section 3, the effect of the chemical field is not even clear. Progress in understanding the thermal state of the inner core will require more constraints on the CMB heat flux and thermophysical parameters of the core and inner core. More constraints on $Q_{\mathrm{cmb}}$ and on the age of the inner core can be expected from seismological observations of post-perovskite lens (Hernlund et al. 2005; Lay et al. 2006; van der Hilst et al. 2007) and an improved understanding of D" dynamics, but the conclusions would still depend heavily on the determination of the thermophysical properties of iron at core conditions. In particular, new experimental or theoretical estimates of the thermal conductivity of iron at Earth's inner core conditions are strongly needed.

We have developed an evolutionary model of inner core convection which is coupled with the core thermal and compositional 
evolution. We have focused on the 'low viscosity limit' of inner core convection which is expected for a viscosity smaller than $\sim 10^{18} \mathrm{~Pa}$ $\mathrm{s}$ (Alboussière et al. 2010). If the viscosity is larger, a translation mode is expected, with associated melting and crystallization (Alboussière et al. 2010; Monnereau et al. 2010). In the low viscosity regime, convection is typical of high Rayleigh number internally driven convection, and can be quite vigorous (convective velocities are expected to be similar to that in the mantle). This type of convection is dominated by small scale motions and is time dependent, and it is not clear how it could produce a large scale N-S cylindrical anisotropy. If the viscosity is small enough or the magnetic field large enough, coupling of convection with the magnetic field diffused in the inner core may plausibly favour large scale axisymmetric motions compatible with the inner core seismic anisotropy, while still allowing for smaller scale flow and heterogeneities.

An interesting output of our work is that several different possible scenarios can result in a layered structure of the inner core, and may provide explanations for the presence of the innermost inner core. If compositional stratification is negligible, a possibility is that the inner core has been convecting early in its history before being stabilized due to the secular decrease of the cooling rate of the core. In this case, the structure of the deep inner core may be interpreted as a frozen-in evidence of this early convective episode [see also Buffett (2009)]. If a significant compositional stratification develops during inner core growth, the convective flow is progressively confined in the deep inner core by the stronger compositional stratification of the outer part of the inner core. It is possible that thermally driven convection remains active in the deep inner core, although convection is expected to be eventually shut off due to the gradual strengthening of the compositional stratification and the decrease in core cooling rate. For each of these scenarios, it is possible to find plausible sets of parameters (inner core age or $Q_{\mathrm{cmb}}$, viscosity, magnitude of the compositional stratification) for which the radius at which convection stops corresponds to the radius of the seismically inferred innermost inner core.

\section{ACKNOWLEDGMENTS}

We thank two anonymous reviewers for their constructive comments. The authors are grateful to Thierry Alboussière for fruitful discussions and encouragement. All the computations presented in this paper were performed at the Service Commun de Calcul Intensif de l'Observatoire de Grenoble (SCCI). R. Deguen was partly supported by grant EAR-0909622 from the Geophysics Program of the National Science Foundation.

\section{REFERENCES}

Alboussière, T., Deguen, R. \& Melzani, M., 2010. Melting induced stratification above the Earth's inner core due to convective translation, Nature, 466, 744-747.

Alfè, D., Gillan, M.J. \& Price, G.D., 2002. Ab initio chemical potentials of solid and liquid solutions and the chemistry of the Earth's core, J. Chem. Phys., 116, 7127-7136.

Allègre, C.J., Poirier, J.-P., Humler, E. \& Hofmann, A.W., 1995. The chemical composition of the Earth, Earth planet. Sci. Lett., 134, 515-526.

Baines, P.G. \& Gill, A.E., 1969. On thermohaline convection with linear gradients, J. Fluid Mech., 37, 289-306.

Beghein, C. \& Trampert, J., 2003. Robust normal mode constraints on innercore anisotropy from model space search, Science, 299, 552-555.

Bergman, M.I., 1997. Measurements of elastic anisotropy due to solidification texturing and the implications for the Earth's inner core, Nature, 389, $60-63$.
Bouhifd, M.A., Gautron, L., Bolfan-Casanova, N., Malavergne, V., Hammouda, T., Andrault, D. \& Jephcoat, A.P., 2007. Potassium partitioning into molten iron alloys at high-pressure: implications for Earth's core, Phys. Earth planet. Inter., 160, 22-33.

Brito, D., Elbert, D. \& Olson, P., 2002. Experimental crystallization of gallium: ultrasonic measurements of elastic anisotropy and implications for the inner core, Phys. Earth planet. Inter., 129, 325-346.

Buffett, B., 2000. Dynamics of the Earth's core, Geophys. Monogr. Ser., 117, $37-62$.

Buffett, B., 2009. Onset and orientation of convection in the inner core, Geophys. J. Int, 179, 711-719.

Buffett, B.A. \& Bloxham, J., 2000. Deformation of Earth's inner core by electromagnetic forces, Geophys. Res. Lett., 27, 4001-4004.

Buffett, B.A. \& Wenk, H.-R., 2001. Texturing of the Earth's inner core by Maxwell stresses, Nature, 413, 60-63.

Buffett, B.A., Huppert, H.E., Lister, J.R. \& Woods, A.W., 1996. On the thermal evolution of the Earth's core, J. geophys. Res., 101, 7989-8006.

Buffett, B.A., Garnero, E.J. \& Jeanloz, R., 2000. Sediments at the top of Earth's core, Science, 290, 1338-1342.

Bukowinski, M.S.T., 1976. The effect of pressure on the physics and chemistry of potassium, Geophys. Res. Lett., 3, 491-494.

Calvet, M., Chevrot, S. \& Souriau, A., 2006. $P$-wave propagation in transversely isotropic media: II. Application to inner core anisotropy: effects of data averaging, parametrization and a priori information, Phys. Earth planet. Inter., 156, 21-40.

Chabot, N.L. \& Drake, M.J., 1999. Crystallization of magmatic iron meteorites: the role of mixing in the molten core, Meteoritics Planet. Sci., 34, 235-246.

Chalmers, B., 1964. Principles of Solidification, J. Wiley \& Sons, New York, NY, 319pp.

Chandrasekhar, S., 1961. Hydrodynamic and Hydromagnetic Stability, International Series of Monographs on Physics, Clarendon, Oxford.

Christensen, U. \& Aubert, J., 2006. Scaling properties of convection-driven dynamos in rotating spherical shells and application to planetary magnetic fields, Geophys. J. Int., 166(1), 97-114.

Corgne, A., Keshav, S., Fei, Y. \& McDonough, W.F., 2007. How much potassium is in the Earth's core? New insights from partitioning experiments, Earth planet. Sci. Lett., 256, 567-576.

Cormier, V.F., 2007. Texture of the uppermost inner core from forward- and back-scattered seismic waves, Earth planet. Sci. Lett., 258, 442-453.

Cormier, V.F. \& Stroujkova, A., 2005. Waveform search for the innermost inner core, Earth planet. Sci. Lett., 236, 96-105.

Crank, J., 1984. Free and Moving Boundary Problems, Clarendon Press, Oxford.

Creager, K.C., 1992. Anisotropy of the inner core from differential travel times of the phases PKP and PKIKP, Nature, 356, 309-314.

Creager, K.C., 1999. Large-scale variations in inner core anisotropy., $J$. geophys. Res., 104, 23 127-23 139.

Deguen, R. \& Cardin, P., 2009. Tectonic history of the Earth's inner core preserved in its seismic structure, Nat. Geosci., 2, 419-422.

Deguen, R., Alboussière, T. \& Brito, D., 2007. On the presence and structure of a mush at the inner core boundary of the Earth, Phys. Earth planet. Inter., 274, 1887-1891.

Dziewonski, A.M. \& Anderson, D.L., 1981. Preliminary reference Earth model, Phys. Earth planet. Inter., 25, 297-356.

Fearn, D., Loper, D. \& Roberts, P., 1981. Structure of the Earth's inner core, Nature, 292, 232-233.

Fearn, D.R. \& Loper, D.E., 1981. Compositional convection and stratification of Earth's core, Nature, 289, 393-394.

Garcia, R., 2002. Constraints on upper inner-core structure from waveform inversion of core phases, Geophys. J. Int., 150, 651-664.

Garcia, R. \& Souriau, A., 2000. Inner core anisotropy and heterogeneity level, Geophys. Res. Lett., 27, 3121-3124.

Gessmann, C.K. \& Wood, B.J., 2002. Potassium in the Earth's core?, Earth planet. Sci. Lett., 200, 63-78.

Gillet, N., Jault, D., Canet, E. \& Fournier, A., 2010. Fast torsional waves and strong magnetic field within the earths core, Nature, 465(7294), 7477. 
Gubbins, D., Alfè, D., Masters, G., Price, G.D. \& Gillan, M., 2004. Gross thermodynamics of two-component core convection, Geophys. J. Int., 157, 1407-1414.

Hernlund, J.W., Thomas, C. \& Tackley, P.J., 2005. A doubling of the postperovskite phase boundary and structure of the Earth's lowermost mantle, Nature, 434, 882-886.

Ishii, M. \& Dziewoński, A.M., 2002. The innermost inner core of the earth: evidence for a change in anisotropic behavior at the radius of about 300 km, Proc. Natl. Acad. Sci., 99, 14 026-14 030.

Jeanloz, R. \& Wenk, H.-R., 1988. Convection and anisotropy of the inner core, Geophys. Res. Lett., 15, 72-75.

Karato, S. I., 1993. Inner core anisotropy due to the magnetic field-induced preferred orientation of iron, Science, 262, 1708-1711.

Karato, S. I., 1999. Seismic anisotropy of the Earth's inner core resulting from flow induced by Maxwell stresses, Nature, 402, 871-873.

Karato, S. I., 2000. Dynamics and anisotropy of the Earth's inner core. Importance of the magnetic coupling with the outer core., Proc. Japan Acad. Ser. B, 76, 1-6.

Labrosse, S., 2003. Thermal and magnetic evolution of the Earth's core, Phys. Earth planet. Inter, 140(1-3), 127-143.

Labrosse, S., Poirier, J.-P. \& Le Mouël, J.-L., 2001. The age of the inner core, Earth planet. Sci. Lett., 190, 111-123.

Lay, T., Hernlund, J., Garnero, E.J. \& Thorne, M.S., 2006. A post-perovskite lens and D" heat flux beneath the central pacific, Science, 314, 1272-1276.

Lay, T., Hernlund, J. \& Buffett, B.A., 2008. Core-mantle boundary heat flow, Nat. Geosci., 1(1), 25-32.

Lee, K.K.M. \& Jeanloz, R., 2003. High-pressure alloying of potassium and iron: Radioactivity in the Earth's core? Geophys. Res. Lett., 30(23), $230000-230001$

Loper, D.E., 1983. Structure of the inner core boundary, Geophys. Astrophys. Fluid Dyn., 25, 139-155.

McKenzie, D., Roberts, J. \& Weiss, N., 1974. Convection in the Earth's mantle: towards a numerical simulation, J. Fluid Mech., 62(03), 465-538.

Monnereau, M., Calvet, M., Margerin, L. \& Souriau, A., 2010. Lopsided growth of Earth's inner core, Science, 328, 1014-1017.

Morelli, A., Dziewonski, A.M. \& Woodhouse, J.H., 1986. Anisotropy of the inner core inferred from PKIKP travel times., Geophys. Res. Lett., 13, $1545-1548$

Murthy, V.R., van Westrenen, W. \& Fei, Y., 2003. Experimental evidence that potassium is a substantial radioactive heat source in planetary cores, Nature, 423, 163-165.

Nimmo, F., 2007. Energetics of the core, in Treatise on Geophysics, Vol. 8, pp. 31-65, ed. Schubert, G., Elsevier, Amsterdam.

Niu, F.L. \& Chen, Q.-F., 2008. Seismic evidence for distinct anisotropy in the innermost inner core., Nat. Geosci., 1, 692-696.

Niu, F.L. \& Wen, L.X., 2001. Hemispherical variations in seismic velocity at the top of the Earth's inner core, Nature, 410, 1081-1084.

Parker, L.J., Atou, T. \& Badding, J.V., 1996. Transition element-like chemistry for potassium under pressure, Science, 273, 95-97.

Parmentier, E.M. \& Sotin, C., 2000. Three-dimensional numerical experiments on thermal convection in a very viscous fluid: implications for the dynamics of a thermal boundary layer at high Rayleigh number, Phys. Fluids, 12, 609-617.

Parmentier, E.M., Sotin, C. \& Travis, B.J., 1994. Turbulent 3-D thermal convection in an infinite Prandtl number, volumetrically heated fluid: implications for mantle dynamics, Geophys. J. Int., 116, 241-251.

Peng, Z., Koper, K., Vidale, J., Leyton, F. \& Shearer, P., 2008. Inner-core fine-scale structure from scattered waves recorded by LASA, J. geophys. Res., 113, 9312-9323.

Poirier, J.-P., 1994. Physical properties of the Earth's core, C.R. Acad. Sci. Paris, 318, 341-350.

Poupinet, G. \& Kennett, B.L.N., 2004. On the observation of high frequency PKiKP and its coda in Australia, Phys. Earth planet. Inter., 146, 497-511.

Poupinet, G., Pillet, R. \& Souriau, A., 1983. Possible heterogeneity of the earth's core deduced from PKIKP travel times, Nature, 305, 204-206.

Shimizu, H., Poirier, J.-P. \& Le Mouël, J.-L., 2005. On crystallization at the inner core boundary, Phys. Earth planet. Inter., 151, 37-51.
Song, X. \& Helmberger, D.V., 1995. Depth dependence of anisotropy of Earth's inner core, J. geophys. Res., 100, 9805-9816.

Souriau, A., 2007. The Earth's cores, in Treatise on Geophysics, Vol. 1, pp. 655-693, ed. Schubert, G., Elsevier, Amsterdam.

Souriau, A. \& Poupinet, G., 1991. The velocity profile at the base of the liquid core from $\mathrm{PKP}(\mathrm{BC}+\mathrm{Cdiff})$ data: an argument in favor of radial inhomogeneity, Geophys. Res. Lett., 18, 2023-2026.

Souriau, A. \& Romanowicz, B., 1996. Anisotropy in inner core attenuation: a new type of data to constrain the nature of the solid core, Geophys. Res. Lett., 23, 1-4.

Souriau, A. \& Romanowicz, B., 1997. Anisotropy in the inner core: relation between P-velocity and attenuation, Phys. Earth planet. Inter., 101, 33-47.

Stacey, F.D., 1995. Theory of thermal and elastic properties of the lower mantle and core, Phys. Earth planet. Inter., 89, 219-245.

Stacey, F.D. \& Anderson, O.L., 2001. Electrical and thermal conductivities of Fe-Ni-Si alloy under core conditions, Phys. Earth planet. Inter., 124, $153-162$.

Stacey, F.D. \& Davis, P.M., 2008. Physics of the Earth, Cambridge University Press, Cambridge.

Sumita, I. \& Bergman, M.I., 2007. Inner-core dynamics, in Treatise on Geophysics, Vol. 8, pp. 299-318, ed. Schubert, G., Elsevier, Amsterdam.

Sumita, I., Yoshida, S., Hamano, Y. \& Kumazawa, M., 1995. A model for the structural evolution of the earth's core and its relation to the observations, in The Earth's Central Part: Its Structure and Dynamics, pp. 232-260, ed. Yukutake, T., Terra Scientific Company, Tokyo.

Sumita, I., Yoshida, S., Kumazawa, M. \& Hamano, Y., 1996. A model for sedimentary compaction of a viscous media and its application to innercore growth, Geophys. J. Int., 124, 302-324.

Sun, X. \& Song, X., 2008a. The inner inner core of the Earth: texturing of iron crystals from three-dimensional seismic anisotropy, Earth planet Sci. Lett., 269, 56-65.

Sun, X. \& Song, X., 2008b. Tomographic inversion for three-dimensional anisotropy of Earth's inner core, Phys. Earth planet. Inter., 167, 53-70.

Takehiro, S., 2010. Fluid motions induced by horizontally heterogeneous Joule heating in the Earth's inner core, Phys. Earth planet. Inter. 184(3-4), 134-142.

Tanaka, S. \& Hamaguchi, H., 1997. Degree one heterogeneity and hemispherical variation of anisotropy in the inner core from PKP(BC)PKP(DF) times, J. geophys. Res., 102, 2925-2938.

Tkalcic, H. \& Kennett, B.L.N., 2008. Core structure and heterogeneity: a seismological perspective, Aust. J. Earth Sci., 55, 419-431.

Tkalčić, H., 2010. Large variations in travel times of mantle-sensitive seismic waves from the south sandwich islands: is the earth's inner core a conglomerate of anisotropic domains?, Geophys. Res. Lett., 37, L14312, doi:10.1029/2010GL043841

Tritton, D., 1988. Physical Fluid Dynamics, Clarendon Press, Oxford.

Tromp, J., 1993. Support for anisotropy of the Earth's inner core from free oscillations., Nature, 366, 678-681.

Turner, J.S., 1980. Buoyancy Effects in Fluids, Cambridge University Press, Cambridge.

van der Hilst, R.D., de Hoop, M.V., Wang, P., Shim, S., Ma, P. \& Tenorio, L., 2007. Seismostratigraphy and thermal structure of Earth's core-mantle boundary region, Science, 315, 1813-1817.

Veronis, G., 1965. On finite amplitude instability in thermohaline convection, J. Marine Res., 23, 1-17.

Vidale, J.E. \& Earle, P.S., 2000. Fine-scale heterogeneity in the Earth's inner core, Nature, 404, 273-275.

Vočadlo, L., 2007. Ab initio calculations of the elasticity of iron and iron alloys at inner core conditions: evidence for a partially molten inner core?, Earth planet. Sci. Lett., 254, 227-232.

Weber, P. \& Machetel, P., 1992. Convection within the inner-core and thermal implications, Geophys. Res. Lett., 19, 2107-2110.

Weinstein, S. \& Olson, P., 1990. Planforms in thermal convection with internal heat sources at large Rayleigh and Prandtl numbers, Geophys. Res. Lett., 17(3), 239-242. 
Wenk, H.-R., Baumgardner, J.R., Lebensohn, R.A. \& Tomé, C.N., 2000. A convection model to explain anisotropy of the inner core, J. geophys. Res., 105, 5663-5678.

Woodhouse, J.H., Giardini, D. \& Li, X.-D., 1986. Evidence for inner core anisotropy from free oscillations., Geophys. Res. Lett., 13, 1549-1552.

Worster, M.G., 1991. Natural convection in a mushy layer, J. Fluid Mech., 224, 335-359.

Yoshida, S., Sumita, I. \& Kumazawa, M., 1996. Growth model of the inner core coupled with the outer core dynamics and the resulting elastic anisotropy, J. geophys. Res., 101, 28085-28104.

Yukutake, T., 1998. Implausibility of thermal convection in the Earth's solid inner core, Phys. Earth planet. Inter., 108, 1-13.

\section{APPENDIX A: COMPOSITIONAL EXPANSION COEFFICIENT}

The chemical expansion coefficient can be estimated from the partial atomic volumes of $\mathrm{Fe}, \mathrm{Si}, \mathrm{S}$ and $\mathrm{O}$ calculated by Alfè et al. (2002). The density of the Fe-Le alloy (where Le stands for 'light element') can be written without loss of generality as

$\rho=\frac{(1-x) m_{\mathrm{Fe}}+x m_{\mathrm{Le}}}{(1-x) v_{\mathrm{Fe}}+x v_{\mathrm{Le}}}$ where $x$ is the mole fraction of light element, $m_{\mathrm{Fe}}=56$ and $m_{\mathrm{Le}}$ are the atomic masses of $\mathrm{Fe}$ and the light element $\left(m_{\mathrm{Si}}=\right.$ $\left.28, m_{\mathrm{S}}=32, m_{\mathrm{O}}=16\right)$, and $v_{\mathrm{Fe}}, v_{\mathrm{Le}}$ are the partial atomic volumes of Fe and the light element in the binary mixture (Alfè et al. 2002). Alfè et al. (2002) found that, in hcp iron at inner core conditions, the partial atomic volumes of $\mathrm{Si}$ and $\mathrm{S}$ are essentially equal to that of $\mathrm{Fe}$ (i.e. $\mathrm{Si}$ and $\mathrm{S}$ atoms replace $\mathrm{Fe}$ atoms in the $h c p$ lattice without significant change in volume), while $v_{\mathrm{O}}$ significantly differs from $v_{\mathrm{Fe}}$. The logarithmic derivative of eq. (A1) gives

$\frac{1}{\rho} \frac{\partial \rho}{\partial x}=\frac{m_{\mathrm{Le}}-m_{\mathrm{Fe}}}{m_{\mathrm{Fe}}+\left(m_{\mathrm{Le}}-m_{\mathrm{Fe}}\right) x}-\frac{v_{\mathrm{Le}}-v_{\mathrm{Fe}}}{v_{\mathrm{Fe}}+\left(v_{\mathrm{Le}}-v_{\mathrm{Fe}}\right) x}$.

$\alpha_{c}$ is given by

$\alpha_{c}=\frac{1}{\rho} \frac{\partial \rho}{\partial c}=\frac{d x}{d c} \frac{1}{\rho} \frac{\partial \rho}{\partial x}=\frac{\left[m_{\mathrm{Fe}}+\left(m_{\mathrm{Le}}-m_{\mathrm{Fe}}\right) x\right]^{2}}{m_{\mathrm{Le}} m_{\mathrm{Fe}}} \frac{1}{\rho} \frac{\partial \rho}{\partial x}$.

With the partial atomic volumes estimated by Alfè et al. (2002), we find $\alpha_{c}^{\mathrm{Si}}=-0.91, \alpha_{c}^{\mathrm{S}}=-0.67$ and $\alpha_{c}^{\mathrm{O}}=-1.3$ in the limit $c \rightarrow 0$. The expansion coefficient depends only weakly on $c$ : for example, $\alpha_{c}^{\mathrm{Si}}=-0.87$ for $c^{\mathrm{Si}}=4.4 \mathrm{wt} . \%$. 